\title{
ADAPTIVE DISCONTINUOUS GALERKIN APPROXIMATIONS TO FOURTH ORDER PARABOLIC PROBLEMS
}

\author{
EMMANUIL H. GEORGOULIS AND JUHA M. VIRTANEN
}

\begin{abstract}
An adaptive algorithm, based on residual type a posteriori indicators of errors measured in $L^{\infty}\left(L^{2}\right)$ and $L^{2}\left(L^{2}\right)$ norms, for a numerical scheme consisting of implicit Euler method in time and discontinuous Galerkin method in space for linear parabolic fourth order problems is presented. The a posteriori analysis is performed for convex domains in two and three space dimensions for local spatial polynomial degrees $r \geq 2$. The a posteriori estimates are then used within an adaptive algorithm, highlighting their relevance in practical computations, by resulting in substantial reduction of computational effort.
\end{abstract}

\section{INTRODUCTION}

Fourth order parabolic equations and corresponding initial-boundary value problems appear in the modelling in areas as diverse as biology, phase-field modelling and image processing to name a few. In most cases of practical interest one has to resort to numerical methods for their solution, due to complex geometry and/or the presence of non-linearities.

During the last five decades, finite element methods (FEMs) have been widely used to numerically solve fourth order elliptic or parabolic problems; see, e.g., 3, 9, 11, 14, 15, 17, 37 and the references therein for earlier works. There are, generally speaking, three families of FEMs developed for fourth order problems: conforming, mixed and non-conforming. The classical conforming methods (see, e.g., [14] and the references therein) require the construction of complicated elements with a number of degrees of freedom devoted to ensuring $C^{1}$-continuity across the element interfaces. This results in limitations in the applicability of conforming methods on general, possibly irregular, meshes [40] and their non-trivial extensions to dimensions three or higher. Mixed methods (see, e.g., [11,15] and the references therein), whereby the fourth order operator is first transformed into a system of second order operators, are widely used in practice, but they require very careful treatment in the imposition of essential and natural boundary conditions. Nonconforming methods for fourth order problems were first presented by [3] and then further developed in [9, 17, 24, 37] and other works. The key idea in non-conforming methods is the use of penalties to ensure convergence into the natural energy space of the variational problem, despite finite element basis functions being either just continuous $\left(C^{0}\right.$-interior penalty procedures; see, e.g., [9, 17] $)$ or completely discontinuous (discontinuous Galerkin interior penalty procedures; see, e.g., 3, 24, 25, 37]).

Received by the editor March 7, 2013 and, in revised form, October 28, 2013.

2010 Mathematics Subject Classification. Primary 65M15, 65M50, 65M60. 
Adaptive FEMs based on a posteriori error estimates has been an active field of research in recent years, especially for second order elliptic and parabolic problems. For second order parabolic problems we refer to the classical works based on duality [18 22] and to the more recent approach based on elliptic reconstruction [29, 33,36$]$. For the case of fourth order elliptic problems a posteriori error estimators and indicators have been developed, e.g., in [1,5, 7, 12, 13, 28, 31, 38, 42. A posteriori bounds and adaptive algorithms for parabolic fourth order problems are far less developed in the literature. For instance, the development of adaptive algorithms based on various types of a posteriori indicators for the Cahn-Hilliard fourth order parabolic problem can be found in 4, 26, 35. Error control for variational methods for fourth order parabolic equations has been predominantly focused to space-discrete mixed or conforming formulations. The recent work [35] deals with goal-oriented error estimation for the fully discrete Cahn-Hilliard problem. Therefore, the development of adaptive algorithms based on a posteriori estimators for fully discrete methods for fourth order parabolic problems is still largely an unexplored area.

Advances in a posteriori error analysis of fully discrete schemes with nonconforming spatial discretizations of second order parabolic problems have been recently presented [23, 29]. In [29], an adaptive algorithm based on the derived a posteriori estimates is also considered. Local residual a posteriori error bounds for semi-discrete conforming and mixed spatial discretizations for the Cahn-Hilliard problem and the Hele-Shaw flow are presented in [26]. Finally, a posteriori error estimates in an $L^{2}\left(H^{2}\right)$-type norm and adaptive algorithms for fully discrete schemes with discontinuous Galerkin methods for fourth order problems are proposed in [43. The derivation of reliability bounds in 43 , is based on the elliptic reconstruction framework of Makridakis and Nochetto [36]; we also refer to [29, 33] for some relevant extensions.

This work is concerned with the derivation of a posteriori error estimates in weaker than $L^{2}\left(H^{2}\right)$-norms and their use within an adaptive algorithm for a class of discontinuous Galerkin interior penalty methods for a fully discrete approximation of the problem:

$$
\begin{aligned}
& u_{t}+\Delta^{2} u=f \quad \text { in } \Omega \times(0, T], \\
& u=\nabla u \cdot \mathbf{n}=0 \quad \text { in } \partial \Omega \times(0, T] \text {, } \\
& u=u_{0} \text { in } \Omega \times\{0\},
\end{aligned}
$$

with $\Omega \subset \mathbb{R}^{d}, d=2,3$ a convex polygonal domain with boundary $\partial \Omega$. More specifically, we derive a posteriori error estimators for the error measured in $L^{\infty}\left(L^{2}\right)$ and $L^{2}\left(L^{2}\right)$ norms for a numerical scheme consisting of discontinuous Galerkin method in space and simple backward-Euler time-stepping for the problem (1)-(3). The a posteriori analysis is performed for convex domains (as is usual for these norms) in two and three space dimensions for local spatial polynomial degrees $r \geq 2$. To enable the optimality of the a posteriori estimators in the $L^{\infty}\left(L^{2}\right)$ and $L^{2}\left(L^{2}\right)$ norms, the elliptic reconstruction framework is employed. Moreover, the $L^{2}\left(L^{2}\right)$-norm analysis employs a special test function construction inspired from the a priori analysis of FEMs for wave problems in [2. Somewhat surprisingly, the use of this special testing, in conjunction with the elliptic reconstruction, results in the derivation of $L^{2}\left(L^{2}\right)$-norm a posteriori estimators via a standard energy argument. The efficiency of the a posteriori estimators is assessed numerically. The reliability bounds are used within two variants of a space-time adaptive algorithm. 
The adaptive algorithm is able to achieve the same error reduction with far fewer degrees of freedom compared to uniform meshes, thereby highlighting the relevance of the derived a posteriori estimates in practical computations. The simple model problem (11)-(3) appears to be sufficient in highlighting some of the challenges in the error estimation and adaptivity of finite element methods for more complex fourth order parabolic problems. It appears that the derived a posteriori bounds and the respective adaptive algorithms can be modified in a straightforward fashion to include the original dG method of Baker [3] and $C^{0}$-interior penalty methods [9,17]. Further, due to the use of elliptic reconstruction, it is possible to incorporate different types of a posteriori bounds for the "elliptic" part of the error, such as the ones presented in 38. We also believe that it is possible to easily extend this approach to derive a posteriori bounds for classical non-conforming spatial discretizations for fourth order problems [5, 12 .

The rest of this work is organized as follows. In Section 2 notation is introduced and some standard results needed in the subsequent analysis are recalled. The discontinuous Galerkin (dG) method for the biharmonic problem, along with the derivation of posteriori error bounds for the $\mathrm{dG}$ approximation of the biharmonic problem in the $L^{2}$ norm are derived in Section 3. The respective fully discrete scheme for the parabolic model problem (11)-(3) is given in Section 4, while Section 5] contains the derivation of residual type a posteriori estimates of errors in $L^{\infty}\left(L^{2}\right)$ and $L^{2}\left(L^{2}\right)$ norms for the fully discrete scheme. The efficiency and reliability of the a posteriori estimators is tested on a range of uniform meshes in Section 6 , The adaptive algorithm utilizing the a posteriori estimates in a series of numerical experiments are also presented in Section 6. Some concluding remarks regarding the results and possible extensions are given in Section 7

\section{Notation AND PRELIMINARIES}

The standard Hilbertian Lebesgue space is denoted by $L^{2}(\omega)$, for a domain $\omega \subset \mathbb{R}^{d},(d=2,3)$, with corresponding inner product $\langle\cdot, \cdot,\rangle_{\omega}$ and norm $\|\cdot\|_{\omega}$; when $\omega=\Omega$, we shall drop the subscript writing $\langle\cdot, \cdot$,$\rangle and \|\cdot\|$, respectively. We also denote by $H^{s}(\omega)$, the standard Hilbertian Sobolev space of index $s \geq 0$ of real-valued functions defined on $\omega \subset \mathbb{R}^{d}$, along with the corresponding norm and seminorm $\|\cdot\|_{s, \omega}$ and $|\cdot|_{s, \omega}$, respectively. For $1 \leq p \leq+\infty$, we also define the spaces $L^{p}\left(0, T, H^{s}(\omega)\right)$, consisting of all measurable functions $v:[0, T] \rightarrow H^{s}(\omega)$, for which

$$
\begin{aligned}
& \|v\|_{L^{p}\left(0, T ; H^{s}(\omega)\right)}:=\left(\int_{0}^{T}\|v(t)\|_{s, \omega}^{p} \mathrm{~d} t\right)^{1 / p}<+\infty, \quad \text { for } \quad 1 \leq p<+\infty, \\
& \|v\|_{L^{\infty}(0, T ; X)}:=\operatorname{ess}_{\sup _{0 \leq t \leq T}}\|v(t)\|_{s, \omega}<+\infty, \text { for } p=+\infty .
\end{aligned}
$$

Let $\mathcal{T}$ be a subdivision of $\Omega$ into disjoint elements $\kappa \in \mathcal{T}$. The subdivision $\mathcal{T}$ is assumed to be shape-regular (see, e.g., p. 124 in [14]) and is constructed via smooth mappings $F_{\kappa}: \hat{\kappa} \rightarrow \kappa$ with uniformly bounded Jacobian throughout the mesh family considered, where $\hat{\kappa}$ is the reference element. The above mappings are assumed to be constructed so as to ensure $\bar{\Omega}=\bigcup_{\kappa \in \mathcal{T}} \kappa$ and that the elemental edges are straight segments (i.e., lines or planes). Note that we also use the expression edge to mean side when $d=3$.

The broken Laplacian, $\Delta_{h} u$, is defined elementwise by $\left.\left(\Delta_{h} u\right)\right|_{\kappa}:=\Delta\left(\left.u\right|_{\kappa}\right)$ for all $\kappa \in \mathcal{T}$. 
For a non-negative integer $r$, we denote by $\mathcal{P}_{r}(\hat{\kappa})$, the set of all polynomials of total degree at most $r$, if $\hat{\kappa}$ is the reference simplex, or of degree at most $r$ in each variable, if $\hat{\kappa}$ is the reference hypercube. We consider the finite element space

$$
S^{r}:=\left\{v \in L^{2}(\Omega):\left.v\right|_{\kappa} \circ F_{\kappa} \in \mathcal{P}_{r}(\hat{\kappa}), \kappa \in \mathcal{T}\right\} .
$$

By $\Gamma$ we denote the union of all $(d-1)$-dimensional element edges associated with the subdivision $\mathcal{T}$, including the boundary. Further, we decompose $\Gamma$ into two disjoint subsets $\Gamma=\partial \Omega \cup \Gamma_{\text {int }}$, where $\Gamma_{\text {int }}:=\Gamma \backslash \partial \Omega$.

For two (generic) elements $\kappa^{+}, \kappa^{-} \in \mathcal{T}$ sharing an edge $e=\kappa^{+} \cap \kappa^{-}$, we define the outward normal unit vectors $\mathrm{n}^{+}$and $\mathrm{n}^{-}$on $e$ corresponding to $\partial \kappa^{+}$and $\partial \kappa^{-}$, respectively. For functions $v: \Omega \rightarrow \mathbb{R}$ and $\mathrm{q}: \Omega \rightarrow \mathbb{R}^{d}$, that may be discontinuous across $\Gamma$, we define the following quantities. For $v^{+}:=\left.v\right|_{e \subset \partial \kappa^{+}}, v^{-}:=\left.v\right|_{e \subset \partial \kappa^{-}}$, $\mathrm{q}^{+}:=\left.\mathrm{q}\right|_{e \subset \partial \kappa^{+}}$, and $\mathrm{q}^{-}:=\left.\mathrm{q}\right|_{e \subset \partial \kappa^{-}}$, we set

$\{v\}:=\frac{1}{2}\left(v^{+}+v^{-}\right),\{\mathrm{q}\}:=\frac{1}{2}\left(\mathrm{q}^{+}+\mathrm{q}^{-}\right), \llbracket v \rrbracket:=v^{+} \mathrm{n}^{+}+v^{-} \mathrm{n}^{-},[\mathrm{q}]:=\mathrm{q}^{+} \cdot \mathrm{n}^{+}+\mathrm{q}^{-} \cdot \mathrm{n}^{-}$.

If $e \in \partial \kappa \cap \partial \Omega$, these definitions are modified to $\{v\}:=v^{+},\{\mathrm{q}\}:=\mathrm{q}^{+}, \llbracket v \rrbracket:=v^{+} \mathrm{n}$, $[\mathrm{q}]:=\mathrm{q}^{+} \cdot \mathrm{n}$. With the above definitions, it is easy to verify the identity

$$
\sum_{\kappa \in \mathcal{T}} \int_{\partial \kappa} v \mathrm{q} \cdot \mathbf{n} \mathrm{d} s=\int_{\Gamma} \llbracket v \rrbracket \cdot\{\mathrm{q}\} \mathrm{d} s+\int_{\Gamma_{\mathrm{int}}}\{v\}[\mathrm{q}] \mathrm{d} s,
$$

with $\mathrm{n}$ denoting the outward normal unit vector on $\partial \kappa$, corresponding to $\kappa$.

We define the element size $h_{\kappa}:=\left(\mu_{d}(\kappa)\right)^{1 / d}$, where $\mu_{d}$ is the $d$-dimensional Lebesgue measure; we collect the element sizes into the elementwise constant function $\mathbf{h}: \Omega \rightarrow \mathbb{R}$, with $\left.\mathbf{h}\right|_{\kappa}=h_{\kappa}, \kappa \in \mathcal{T}$ and $\mathrm{h}=\{\mathrm{h}\}$ on $\Gamma$. Also, for two (generic) elements $\kappa^{+}, \kappa^{-}$sharing an edge $e:=\partial \kappa^{+} \cap \partial \kappa^{-} \subset \Gamma_{\text {int }}$, we define $h_{e}:=\mu_{d-1}(e)$.

As we shall be dealing with mesh adaptive algorithms below, we assume that all sequences of meshes considered in this work are locally quasi-uniform, i.e., there exists constant $c \geq 1$, independent of $\mathbf{h}$, such that, for any pair of elements $\kappa^{+}$and $\kappa^{-}$in $\mathcal{T}$ which share an edge, we get

$$
c^{-1} \leq h_{\kappa^{+}} / h_{\kappa^{-}} \leq c .
$$

Finally, we recall a series of some (standard) results used throughout this work; their proofs can be found, e.g., in [6, 8, 10, 14, 16,

Lemma 2.1 (approximation property). Let $0 \leq m \leq r+1$ and $\mathcal{T}$ be a subdivision of $\Omega, \Omega \subset \mathbb{R}^{d}$. Then there exists a constant $C_{\text {app }}$, independent of $h_{\kappa}$, such that for any $u \in H^{m}(\Omega)$ and $\kappa \in \mathcal{T}$, there exists $p: C(\kappa) \rightarrow \mathbb{R}$, with $p \circ F_{\kappa} \in \mathcal{P}_{r}(\kappa)$ and

$$
|u-p|_{j, \kappa} \leq C_{a p p} h_{\kappa}^{m-j}|u|_{m, \kappa}, 0 \leq j \leq m .
$$

Lemma 2.2 (inverse estimate). There exists a constant $C_{\text {inv }}$, independent of $h_{\kappa}$, such that

$$
|p|_{j, \kappa} \leq C_{i n v} h_{\kappa}^{i-j}|p|_{i, \kappa}, 0 \leq i \leq j \leq 2,
$$

for all $p: C(\kappa) \rightarrow \mathbb{R}$, with $p \circ F_{\kappa} \in \mathcal{P}_{r}(\kappa)$.

Lemma 2.3 (trace inequality). For every $u \in H^{1}(\kappa)$, with $\kappa \in \mathcal{T}$, there exists a constant $C_{t r}>0$ independent of $h_{\kappa}$ such that

$$
\|u\|_{0, \partial \kappa}^{2} \leq C_{t r}\left(h_{\kappa}^{-1}|| u \|_{0, \kappa}^{2}+h_{\kappa}|u|_{1, \kappa}^{2}\right) .
$$


Lemma 2.4 (Poincaré-Friedrichs inequality [10]). There exists a constant $C_{p f}$, independent of $h_{\kappa}$, such that for any $u \in L^{2}(\Omega)$, with $\left.u\right|_{\kappa} \in H^{2}(\kappa)$ for all $\kappa \in \mathcal{T}$, we have

$$
\|u\|_{0, \Omega}^{2}+|u|_{1, \Omega}^{2} \leq C_{p f}\left(|u|_{2, \Omega}^{2}+\left\|h^{-3 / 2} \llbracket u \rrbracket\right\|_{0, \Gamma}^{2}+\left\|h^{-1 / 2}[\nabla u]\right\|_{0, \Gamma}^{2}\right) .
$$

\section{Discontinuous Galerkin method for the Biharmonic PRoblem}

We consider the biharmonic equation

$$
\Delta^{2} \tilde{u}=\phi \quad \text { in } \Omega
$$

with homogeneous essential boundary conditions

$$
\tilde{u}=0, \quad \nabla \tilde{u} \cdot \mathrm{n}=0 \quad \text { on } \partial \Omega,
$$

where $\mathrm{n}$ denotes the unit outward normal vector to $\partial \Omega$ and $\phi \in L^{2}(\Omega)$. Then the regularity of the problem implies that $\tilde{u} \in H^{4}(\Omega) \cap H_{0}^{2}(\Omega) 30$.

Upon defining the lifting operator $\mathcal{L}: \mathcal{S}:=S^{r}+H_{0}^{2}(\Omega) \rightarrow S^{r}$ by

$$
\int_{\Omega} \mathcal{L}(\nu) \psi \mathrm{d} x=\int_{\Gamma}(\llbracket \nu \rrbracket \cdot\{\nabla \psi\}-\{\psi\}[\nabla \nu]) \mathrm{d} s \quad \forall \psi \in S^{r},
$$

the (symmetric) interior penalty discontinuous Galerkin (dG) method for (12), (13) is given by:

$$
\text { find } \tilde{u}_{h} \in S^{r} \text { such that } B\left(\tilde{u}_{h}, v_{h}\right)=l\left(v_{h}\right) \quad \forall v_{h} \in S^{r} \text {, }
$$

where the bilinear form $B: \mathcal{S} \times \mathcal{S} \rightarrow \mathbb{R}$ and the linear form $l: \mathcal{S} \rightarrow \mathbb{R}$ are given by

$$
B(w, v):=\int_{\Omega}\left(\Delta_{h} w \Delta_{h} v+\mathcal{L}(w) \Delta_{h} v+\Delta_{h} w \mathcal{L}(v)\right) \mathrm{d} x+B_{p}(w, v)
$$

with

and

$$
B_{p}(w, v):=\int_{\Gamma}(\sigma \llbracket w \rrbracket \cdot \llbracket v \rrbracket+\xi[\nabla w][\nabla v]) \mathrm{d} s,
$$

respectively, for $w, v \in \mathcal{S}$. The piecewise constant discontinuity penalization parameters $\sigma, \xi: \Gamma \rightarrow \mathbb{R}$ are given by

$$
\left.\sigma\right|_{e}=\sigma_{0}\left(\left.\mathrm{~h}\right|_{e}\right)^{-3},\left.\quad \xi\right|_{e}=\xi_{0}\left(\left.\mathrm{~h}\right|_{e}\right)^{-1},
$$

respectively, where $\sigma_{0}>0$ and $\xi_{0}>0$. To guarantee the stability of the IPDG method defined in (15), $\sigma_{0}$ and $\xi_{0}$ must be selected sufficiently large.

Note that this formulation is inconsistent for trial and test functions belonging to the solution space $\mathcal{S}$. However, when $w, v \in S^{r}$, in view of (14), (16) gives

$$
\begin{aligned}
B(w, v)= & \int_{\Omega} \Delta_{h} w \Delta_{h} v \mathrm{~d} x+\int_{\Gamma}(\{\nabla \Delta w\} \cdot \llbracket v \rrbracket+\{\nabla \Delta v\} \cdot \llbracket w \rrbracket \\
& -\{\Delta w\}[\nabla v]-\{\Delta v\}[\nabla w]+\sigma \llbracket w \rrbracket \cdot \llbracket v \rrbracket+\xi[\nabla w][\nabla v]) \mathrm{d} s
\end{aligned}
$$

therefore, (15) coincides with the symmetric version interior penalty method presented in [41. For the bilinear form $B(\cdot, \cdot)$ in (16) we have the continuity and coercivity with respect to the energy norm on $\mathcal{S}$ defined by

$$
\||w|\|=\left(\left\|\Delta_{h} w\right\|_{\Omega}^{2}+\|\sqrt{\sigma} \llbracket w \rrbracket\|_{\Gamma}^{2}+\|\sqrt{\xi}[\nabla w]\|_{\Gamma}^{2}\right)^{\frac{1}{2}} .
$$


Lemma 3.1 ([27]). For sufficiently large $\sigma_{0}>0$ and $\xi_{0}>0$ there exist positive constants $C_{\text {cont }}$ and $C_{\text {coer }}$, depending only on the mesh parameters such that

$$
\begin{gathered}
|B(u, v)| \leq C_{\text {cont }}|\|u|\||\|v\||\| \forall u, v \in \mathcal{S} \text { and } \\
B(u, u) \geq C_{\text {coer }}|\|u\||^{2} \forall u \in \mathcal{S} .
\end{gathered}
$$

An a posteriori bound for the energy norm error of the dG method (15) for (12) - (13) has been considered in [28. Now, we shall present an a posteriori bound for the $L^{2}$-norm error (cf. 39] for a corresponding result for the second order problem).

Theorem 3.2 ( $L^{2}$-a posteriori bounds for the elliptic problem). Let $\tilde{u} \in H^{4}(\Omega) \cap$ $H_{0}^{2}(\Omega)$ be the solution of (12) -(13) and let $\tilde{u}_{h} \in S^{r}$ be the $d G$ approximation (15) associated with the mesh $\mathcal{T}$. Then, there exists a positive constant $C_{[3.2]}$, independent of $\mathcal{T}, h, \tilde{u}$ and $\tilde{u}_{h}$, such that

$$
\left\|\tilde{u}-\tilde{u}_{h}\right\| \leq \mathcal{E}\left(\mathcal{T}, \tilde{u}_{h}, \phi\right),
$$

where

$$
\begin{aligned}
\mathcal{E}\left(\mathcal{T}, \tilde{u}_{h}, \phi\right):= & C_{\underline{\text { B.2 }}}\left(\left\|h^{4-\lambda / 2}\left(\phi-\Delta_{h}^{2} \tilde{u}_{h}\right)\right\|^{2}\right. \\
& +\left\|h^{(7-\lambda) / 2}\left[\nabla \Delta \tilde{u}_{h}\right]\right\|_{\Gamma_{\mathrm{int}}}^{2}+\left\|h^{(5-\lambda) / 2} \llbracket \Delta \tilde{u}_{h} \rrbracket\right\|_{\Gamma_{\mathrm{int}}}^{2} \\
& +\sum_{e \in \Gamma}\left(h_{e}^{3-\lambda}\left(1+\xi_{0}^{2}\right)\left\|\left[\nabla \tilde{u}_{h}\right]\right\|^{2}+h_{e}^{1-\lambda}\left(1+\sigma_{0}^{2}\right)\left\|\llbracket \tilde{u}_{h} \rrbracket\right\|^{2}\right)
\end{aligned}
$$

and $\lambda:=2(2-\min \{2, r-1\})$.

Proof. The dual problem

$$
\Delta^{2} z=\tilde{u}-\tilde{u}_{h}=: \tilde{e} \quad \text { in } \Omega,
$$

with homogeneous essential boundary conditions $z=\nabla \tilde{u} \cdot \mathrm{n}=0$ on $\partial \Omega$ clearly satisfies $\tilde{e} \in L^{2}(\Omega)$ and, therefore, the following regularity estimate holds:

$$
\|z\|_{4, \Omega} \leq C_{\mathrm{reg}}\|\tilde{e}\| .
$$

Using (25), integrating by parts twice, applying (6) and (14) as well as the regularity of the dual solution, $z \in H^{4}(\Omega)$, we have

$$
\|\tilde{e}\|^{2}=\sum_{\kappa \in \mathcal{T}} \int_{\kappa} \Delta^{2} z \tilde{e} \mathrm{~d} x=\int_{\Omega} \Delta_{h} z \Delta_{h} \tilde{e} \mathrm{~d} x-\int_{\Gamma}[\nabla \tilde{e}]\{\Delta z\} \mathrm{d} s+\int_{\Gamma} \llbracket \tilde{e} \rrbracket \cdot\{\nabla \Delta z\} \mathrm{d} s .
$$

By using the fact that $\tilde{u}$ is a weak solution and integrating the term involving $\Delta_{h} z \Delta_{h} \tilde{u}_{h}$ by parts, we arrive at,

$$
\begin{aligned}
\|\tilde{e}\|^{2}= & B(\tilde{u}, z)-\int_{\Omega} \Delta_{h} z \Delta_{h} \tilde{u}_{h} \mathrm{~d} x+\int_{\Gamma}\left[\nabla \tilde{u}_{h}\right]\{\Delta z\} \mathrm{d} s-\int_{\Gamma} \llbracket \tilde{u}_{h} \rrbracket \cdot\{\nabla \Delta z\} \mathrm{d} s \\
= & l(z)-\int_{\Omega} z \Delta^{2} \tilde{u}_{h} \mathrm{~d} x+\int_{\Gamma_{\mathrm{int}}}\{z\}\left[\nabla \Delta_{h} \tilde{u}_{h}\right] \mathrm{d} s-\int_{\Gamma_{\mathrm{int}}}\{\nabla z\} \cdot \llbracket \Delta_{h} \tilde{u}_{h} \rrbracket \mathrm{d} s \\
& +\int_{\Gamma}\left[\nabla \tilde{u}_{h}\right]\{\Delta z\} \mathrm{d} s-\int_{\Gamma} \llbracket \tilde{u}_{h} \rrbracket \cdot\{\nabla \Delta z\} \mathrm{d} s .
\end{aligned}
$$


Using the standard orthogonal $L^{2}$ projection, $\Pi: \mathcal{S} \rightarrow S^{r}$, of $z$, we can derive the following identity by integrating by parts and using (6) and (14) as follows:

(29)

$$
\begin{aligned}
0=l( & -\Pi z)-B\left(\tilde{u}_{h},-\Pi z\right) \\
= & \sum_{\kappa \in \mathcal{T}} \int_{\kappa}\left(\left(\phi-\Delta^{2} \tilde{u}_{h}\right)(-\Pi z)-\mathcal{L}\left(\tilde{u}_{h}\right) \Delta_{h}(-\Pi z)\right) \mathrm{d} x+\int_{\Gamma_{\text {int }}}\{-\Pi z\}\left[\nabla \Delta_{h} \tilde{u}_{h}\right] \mathrm{d} s \\
& -\int_{\Gamma_{\text {int }}}\{\nabla(-\Pi z)\} \cdot \llbracket \Delta_{h} \tilde{u}_{h} \rrbracket \mathrm{d} s-\int_{\Gamma}\left(\sigma \llbracket \tilde{u}_{h} \rrbracket \cdot \llbracket-\Pi z \rrbracket+\xi\left[\nabla \tilde{u}_{h}\right][\nabla(-\Pi z)]\right) \mathrm{d} s .
\end{aligned}
$$

Using (14) in (29) and combining (28) and (29), we get

(30)

$$
\begin{aligned}
\|\tilde{e}\|^{2}= & \|\tilde{e}\|^{2}+l(-\Pi z)-B\left(\tilde{u}_{h},-\Pi z\right) \\
= & \int_{\Omega}\left(\phi-\Delta_{h}^{2} \tilde{u}_{h}\right)(z-\Pi z) \mathrm{d} x+\int_{\Gamma_{\mathrm{int}}}\left(\{z-\Pi z\}\left[\nabla \Delta \tilde{u}_{h}\right] \mathrm{d} s-\llbracket \Delta \tilde{u}_{h} \rrbracket \cdot\{\nabla(z-\Pi z)\}\right) \mathrm{d} s \\
& -\int_{\Gamma} \llbracket \tilde{u}_{h} \rrbracket \cdot\left(\{\nabla \Delta(z-\Pi z)\}+\sigma_{0} \mathrm{~h}^{-3} \llbracket z-\Pi z \rrbracket\right) \mathrm{d} s \\
& +\int_{\Gamma}\left[\nabla\left(\tilde{u}_{h}\right)\right]\left(\{\Delta(z-\Pi z)\}+\xi_{0} \mathrm{~h}^{-1}[\nabla(z-\Pi z)]\right) \mathrm{d} s .
\end{aligned}
$$

The assertion then follows by applying Young's inequality, the trace inequality (10) where appropriate, the approximation property (8) and the regularity of the dual problem on each of the terms on the right-hand side of (30).

Remark 3.3. If a smooth $C^{1}$ subspace of the finite element space exists, such as Argyris elements in two dimensions, or corresponding constructions in three dimensions, it is possible to establish an a posteriori $L^{2}$ bound without dependence on penalty parameters; indeed, these terms would vanish from (30) if the projection, $\Pi$, could be defined onto the smooth subspace of $S^{r}$.

Remark 3.4. It is interesting to note that the a posteriori error bound of (23) reflects the suboptimal $L^{2}$-norm error convergence of the dG method when quadratic polynomials are applied. Similar behaviour is observed theoretically and numerically in 27] and in [41] in the context of the a priori error analysis of the same method.

\section{DG METHOD FOR THE PARABOLIC PROBLEM}

Throughout the remainder of this work, we shall denote by $u$ the weak solution of the problem (11)-(3) in variational form: find $u \in H^{1}\left(0, T ; H^{4}(\Omega) \cap H_{0}^{2}(\Omega)\right)$ such that

$$
\begin{aligned}
\left\langle u_{t}, \phi\right\rangle+B(u, \phi) & =\langle f, \phi\rangle \quad \forall \phi \in H_{0}^{2}(\Omega), \\
u & =u_{0} \in L^{2}(\Omega) \quad \text { in } \Omega \times\{0\} .
\end{aligned}
$$

We consider a subdivision of the time interval $(0, T]$ to be the family of intervals $\left\{\left(t^{n-1}, t^{n}\right] ; n=1, \ldots, N\right.$, with $t^{0}=0, t^{n-1} \leq t^{n}$ and $\left.t^{N}=T\right\}$, with local timestep $\lambda_{n}:=t^{n}-t^{n-1}$. Associated with this time-subdivision, let $\mathcal{T}_{n}, n=0, \ldots, N$, be a sequence of meshes which are assumed to be compatible, in the sense that for any two consecutive meshes $\mathcal{T}_{n-1}$ and $\mathcal{T}_{n}, \mathcal{T}_{n}$ can be obtained from $\mathcal{T}_{n-1}$ by locally coarsening some of its elements and then locally refining some (possibly other) elements. The finite element space corresponding to $\mathcal{T}_{n}$ will be denoted by $S^{r, n}$ 
and the respective $\mathrm{dG}$ bilinear form by $B^{n}(\cdot, \cdot)$. The backward Euler-dG method for approximating (31) is then given by: for each $n=1, \ldots, N$, find

$$
U^{n} \in S^{r, n} \text { such that }\left\langle\frac{U^{n}-U^{n-1}}{\lambda_{n}}, V\right\rangle+B^{n}\left(U^{n}, V\right)=\left\langle\tilde{f}^{n}, V\right\rangle \quad \forall V \in S^{r, n},
$$

where $\tilde{f}^{0}(\cdot):=f(\cdot, 0)$ and $\tilde{f}^{n}(\cdot)$ for $n=1, \ldots, N$ is a piecewise polynomial of degree $p$ in time $L^{2}$ projection in time of the source function $f$. In practice, it suffices to take $p=0$ to achieve a first-order-in-time convergent method. We also set $U^{0}:=\Pi^{0} u_{0}$, with $\Pi^{0}: L^{2}(\Omega) \rightarrow S^{r, 0}$ is the orthogonal $L^{2}$-projection operator onto the finite element space $S^{r, 0}$.

\section{A POSTERIORI BOUNDS FOR THE PARABOLIC PROBLEM}

We shall derive a posteriori error bounds for the backward Euler-dG method (32) measured in $L^{\infty}\left(L^{2}\right)$ and $L^{2}\left(L^{2}\right)$ norms. To this end, we shall employ an energy argument (with carefully defined test functions) in conjunction with the elliptic reconstruction technique 29, 33, 36.

We begin by extending the sequence $\left\{U^{n}\right\}_{n=1, \ldots, N}$ of numerical solutions into a continuous piecewise linear function of time

$$
U(0)=\Pi^{0} u_{0} \quad \text { and } \quad U(t):=\frac{t-t^{n-1}}{\lambda_{n}} U^{n}+\frac{t^{n}-t}{\lambda_{n}} U^{n-1}
$$

for $t \in\left(t_{n-1}, t_{n}\right]$ and $n=1, \ldots, N$. Further, the discrete elliptic operator $A^{n}$ : $S^{r, n} \rightarrow S^{r, n}$ is defined by:

$$
\text { for } \phi \in S^{r, n}, \quad\left\langle A^{n} \phi, \chi\right\rangle=B^{n}(\phi, \chi) \quad \forall \chi \in S^{r, n} .
$$

We now give definitions of the estimators involved in the estimation of the parabolic part of the error. Estimators at time-step $n$ are denoted by $\infty, n$ subscript and $2, n$ subscript will be used for the cases of $L^{\infty}\left(L^{2}\right)$ and $L^{2}\left(L^{2}\right)$ bounds presented below, respectively.

Definition 5.1 (estimators for the parabolic error). We define the coarsening or mesh-change estimators by

$$
\begin{aligned}
\gamma_{\infty, n} & :=\frac{1}{\lambda_{n}}\left\|\left(I-\Pi^{n}\right) U^{n-1}\right\|^{2} \\
\gamma_{2, n} & :=\left\|\left(I-\Pi^{n}\right) U^{n-1}\right\|^{2}+\sum_{i=1}^{n-1}\left\|\left(\Pi^{i}-\Pi^{i-1}\right) U^{i-1}\right\|^{2},
\end{aligned}
$$

the time-error evolution estimators by

$$
\eta_{\infty, n}:=\left\|g^{n}-g^{n-1}\right\|^{2} \lambda_{n}, \quad \eta_{2, n}:=\left\|g^{n}-g^{n-1}\right\|^{2} \lambda_{n}^{2}+\sum_{i=1}^{n-1} \lambda_{i}^{2}\left\|g^{i}-g^{i-1}\right\|^{2},
$$

$g^{n}:=A^{n} U^{n}-\Pi^{n} \tilde{f}^{n}+\tilde{f}^{n}$; the data approximation error in time estimators by

$$
\beta_{\infty, n}:=\int_{t^{n-1}}^{t^{n}}\left\|\tilde{f}^{n}-f\right\|^{2} \mathrm{~d} t, \quad \beta_{2, n}:=\lambda_{n} \int_{t^{n-1}}^{t^{n}}\left\|\tilde{f}^{n}-f\right\|^{2} \mathrm{~d} t
$$

and an additional space estimator given by

$$
\tilde{\eta}_{\infty, n}:=\mathcal{E}\left(\hat{\mathcal{T}}_{n}, U^{n}-U^{n-1}, g^{n}-g^{n-1}\right)^{2},
$$


where $\hat{\mathcal{T}}_{n}:=\mathcal{T}_{n} \cap \mathcal{T}_{n-1}$ is the finest common coarsening of $\mathcal{T}_{n}$ and $\mathcal{T}_{n-1}$ for each $n=1, \ldots, N$.

Using the notation above, we are ready to state the main result.

Theorem 5.2 (a posteriori bound). Let $u \in L^{2}\left(0, T ; H^{4}(\Omega) \cap H_{0}^{2}(\Omega)\right)$ be the solution of (31) and let $U$ be the approximation obtained by the $d G$ method (32) and defined by (33). Then there exist positive constants $C_{\infty}$ and $C_{2}$, independent of $\mathcal{T}_{n}$, $h, u$ and $U$, for any $n=1, \ldots, N$ such that

$$
\begin{aligned}
& \|e\|_{L^{\infty}\left(0, T ; L^{2}(\Omega)\right)} \leq C_{\infty}\left(\|e(0)\|+\left(\sum_{n=1}^{N}\left(\gamma_{\infty, n}+\eta_{\infty, n}+\beta_{\infty, n}\right) \lambda_{n}\right)^{\frac{1}{2}}\right. \\
& \left.+\left(\sum_{n=1}^{N} \tilde{\eta}_{\infty, n}\right)^{\frac{1}{2}}+\max _{0 \leq n \leq N}\left\{\mathcal{E}\left(\mathcal{T}_{n}, U^{n}, g^{n}\right)\right\}\right), \\
& \|e\|_{L^{2}\left(0, T ; L^{2}(\Omega)\right)} \leq C_{2}\left(\|e(0)\|+\left(\sum_{n=1}^{N}\left(\gamma_{2, n}+\eta_{2, n}+\beta_{2, n}\right) \lambda_{n}\right)^{\frac{1}{2}}\right. \\
& \left.+\left(\sum_{n=1}^{N} \mathcal{E}\left(\mathcal{T}_{n}, U^{n}, g^{n}\right)^{2} \lambda_{n}\right)^{\frac{1}{2}}\right)
\end{aligned}
$$

The proof of this theorem will be the content of the remainder of this section, split into a number of intermediate results.

We begin by defining the elliptic reconstruction $\omega^{n} \in H_{0}^{2}(\Omega)$, of $U^{n}$ to be the solution of the elliptic problem

$$
B^{n}\left(\omega^{n}, v\right)=\left\langle g^{n}, v\right\rangle \quad \forall v \in H_{0}^{2}(\Omega)
$$

where, as above, $g^{n}:=A^{n} U^{n}-\Pi^{n} \tilde{f}^{n}+\tilde{f}^{n}$. We note that under the assumptions on the domain $\Omega$, we also have $\omega^{n} \in H^{4}(\Omega)$. We also extend the elliptic reconstruction into a continuous piecewise linear-in-time function

$$
\omega(t):=\frac{t-t^{n-1}}{\lambda_{n}} \omega^{n}+\frac{t^{n}-t}{\lambda_{n}} \omega^{n-1}
$$

for $t \in\left(t_{n-1}, t_{n}\right]$ and $n=1, \ldots, N$. Finally, we introduce the error decomposition

$$
e:=U-u=\rho-\epsilon, \text { where } \epsilon:=\omega-U, \text { and } \rho:=\omega-u,
$$

where $\rho$ and $\epsilon$ are understood as the parabolic and elliptic error, respectively, and we set $\epsilon_{n}:=\epsilon\left(t_{n}\right)$. The key idea here is that the elliptic part of the error can be estimated using the a posteriori error bounds for the elliptic problem and, furthermore, the parabolic part of the error can be bound by the elliptic part of the error and other known a posteriori terms. See [29, 33, 36] for further discussion on this type of error decomposition.

Lemma 5.3 (error identity). For all $t \in\left(t_{n-1}, t_{n}\right], n=1,2, \ldots, N$, we have

$$
\left\langle\rho_{t}, v\right\rangle+B^{n}(\rho, v)=\left\langle\epsilon_{t}, v\right\rangle+\left\langle\left(I-\Pi^{n}\right) U_{t}, v\right\rangle+\frac{t-t^{n}}{\lambda_{n}}\left\langle g^{n}-g^{n-1}, v\right\rangle+\left\langle\tilde{f}^{n}-f, v\right\rangle,
$$

for any $v \in H_{0}^{2}(\Omega)$, with $I$ denoting the identity mapping. 
Proof. First, from (41) and (34) we have

$$
B^{n}\left(\omega^{n}, v\right)-\left\langle\tilde{f}^{n}, v\right\rangle=B^{n}\left(U^{n}, \Pi^{n} v\right)-\left\langle\Pi^{n} \tilde{f}^{n}, \Pi^{n} v\right\rangle .
$$

Also, using the method (32) and the definition of the $L^{2}$ projection we deduce (46)

$\left\langle U_{t}, v\right\rangle=\left\langle\left(I-\Pi^{n}\right) U_{t}, v\right\rangle+\left\langle U_{t}, \Pi^{n} v\right\rangle=\left\langle\left(I-\Pi^{n}\right) U_{t}, v\right\rangle-\left(B^{n}\left(U_{n}, \Pi^{n} v\right)-\left\langle\Pi^{n} \tilde{f}^{n}, \Pi^{n} v\right\rangle\right)$.

For the elliptic reconstruction error we also have,

$$
B^{n}\left(\omega-\omega^{n}, v\right)=\frac{t-t^{n}}{\lambda_{n}}\left\langle g^{n}-g^{n-1}, v\right\rangle .
$$

Last, for the terms on the left-hand side of (44), we compute

$$
\begin{aligned}
\left\langle e_{t}, v\right\rangle+B^{n}(\rho, v) & =\left\langle U_{t}, v\right\rangle+B^{n}(\omega, v)-\left(\left\langle u_{t}, v\right\rangle+B^{n}(u, v)\right) \\
& =\left\langle U_{t}, v\right\rangle+B^{n}(\omega, v)-\langle f, v\rangle .
\end{aligned}
$$

Using (45), (45), and (47) in (48), along with the identity $e=\rho-\epsilon$ completes the proof.

The a posteriori bounds (39) and (40) will be derived by selecting special test functions $v$ in the energy identity (44) above, along with the estimation of the terms on the right-hand side of (44). More specifically, we consider the following two test functions, $\tilde{v}:=\rho$ for the $L^{\infty}\left(L^{2}\right)$ case, and

$$
\bar{v}(t, \cdot):=\int_{t}^{T} \rho(s, \cdot) \mathrm{d} s, \quad t \in[0, T],
$$

for the $L^{2}\left(L^{2}\right)$ case; this choice is motivated by Baker 2, who used a similar construction for the proof of a priori bounds for the second order wave problem. The latter test function has most notably the following properties:

$$
\begin{aligned}
& \bar{v} \in H^{4}(\Omega) \cap H_{0}^{2}(\Omega) \quad \text { as } \rho \in H^{4}(\Omega) \cap H_{0}^{2}(\Omega) \text { a.e. in }[0, T], \\
& \bar{v}(T, \cdot)=0=\Delta \bar{v}(T, \cdot), \quad \nabla \bar{v}(T, \cdot)=0, \\
& \bar{v}_{t}(t, \cdot)=-\rho(t, \cdot), \quad \text { a.e. in }[0, T] .
\end{aligned}
$$

Next, we consider two auxiliary functions which are needed in the consequent proofs. More specifically, on each interval $t \in\left(t_{n-1}, t_{n}\right]$, for $n=1, \ldots, N$, we define

$$
\tilde{G}(t):=\left(I-\Pi^{n}\right) U+\psi^{n} \quad \text { with } \quad \psi^{n}:=-\left(I-\Pi^{n}\right) U^{n-1}+\psi^{n-1}, \quad \psi^{0}:=0,
$$

and

$$
G(t):=\frac{\lambda_{n}}{2}\left(\frac{t-t^{n}}{\lambda_{n}}\right)^{2}\left(g^{n}-g^{n-1}\right)+\theta^{n} \text { with } \theta^{n}:=-\frac{\lambda_{n}}{2}\left(g^{n}-g^{n-1}\right)+\theta^{n-1}, \quad \theta^{0}=0 .
$$

We note that, for each $n=1, \ldots, N$, we then have $\tilde{G}\left(t^{n}\right)=\psi^{n}, G\left(t^{n}\right)=\theta^{n}$,

$$
\tilde{G}_{t}(t):=\left(I-\Pi^{n}\right) U_{t} \quad \text { and } \quad G_{t}(t):=\frac{t-t^{n}}{\lambda_{n}}\left(g^{n}-g^{n-1}\right) .
$$

The following estimates will be used in the proof of Theorem 5.2 
Lemma 5.4. Let $\tau \in(0, T]$. Then, we have

$$
\begin{aligned}
\int_{0}^{\tau}\left\langle\tilde{G}_{t}, \rho\right\rangle \mathrm{d} t & \leq \sum_{n=1}^{N}\left\|\left(\Pi^{n}-I\right) U^{n-1}\right\| \max _{0 \leq t \leq T}\|\rho\|, \\
\int_{0}^{\tau}\left\langle G_{t}, \rho\right\rangle \mathrm{d} t & \leq \sum_{n=1}^{N} \lambda_{n}\left\|g^{n}-g^{n-1}\right\| \max _{0 \leq t \leq T}\|\rho\|, \\
\int_{0}^{\tau}\left\langle\tilde{f}^{n}-f, \rho\right\rangle \mathrm{d} t & \leq \int_{0}^{\tau}\left\|\tilde{f}^{n}-f\right\| \mathrm{d} t \max _{0 \leq t \leq T}\|\rho\| .
\end{aligned}
$$

Proof. The proofs of these estimates are immediate via Cauchy-Schwarz-in-space and Hölder-in-time inequalities.

In the following three lemmas, we prove bounds for the corresponding terms to the ones in Lemma 5.4 when testing with $\bar{v}$ given in (49).

Lemma 5.5. With the above notation, we have

$$
\begin{gathered}
\sum_{n=1}^{N} \int_{t^{n-1}}^{t^{n}}\left\langle\left(I-\Pi^{n}\right) U_{t}, \bar{v}\right\rangle \mathrm{d} t \leq \sum_{n=1}^{N}\left(\lambda_{n}\left\|\left(I-\Pi^{n}\right) U^{n-1}\right\|^{2}\right. \\
\left.\quad+\lambda_{n}\left\|\sum_{i=1}^{n-1}\left(\Pi^{i}-\Pi^{i-1}\right) U^{i-1}\right\|^{2}\right)^{\frac{1}{2}}\left(\int_{t^{n-1}}^{t^{n}}\|\rho\|^{2} \mathrm{~d} t\right)^{\frac{1}{2}} .
\end{gathered}
$$

Proof. Recalling the definition of $\tilde{G}$, an integration by parts with respect to time gives

$$
\begin{aligned}
\sum_{n=1}^{N} \int_{t^{n-1}}^{t^{n}}\left\langle\left(I-\Pi^{n}\right) U_{t}, \bar{v}\right\rangle \mathrm{d} t & =\sum_{n=1}^{N}[\langle\tilde{G}(t), \bar{v}(t)\rangle]_{t^{n-1}}^{t^{n}}+\sum_{n=1}^{N} \int_{t^{n-1}}^{t^{n}}\left\langle\tilde{G},-\bar{v}_{t}\right\rangle \mathrm{d} t \\
& =\sum_{n=1}^{N} \int_{t^{n-1}}^{t^{n}}\left\langle\tilde{G},-\bar{v}_{t}\right\rangle \mathrm{d} t .
\end{aligned}
$$

We recall the properties of $\bar{v}$ in (50) and we estimate the right-hand term further:

$$
\sum_{n=1}^{N} \int_{t^{n-1}}^{t^{n}}\left\langle\tilde{G},-\bar{v}_{t}\right\rangle \mathrm{d} t \leq \sum_{n=1}^{N}\left(\int_{t^{n-1}}^{t^{n}}\|\tilde{G}\|^{2} \mathrm{~d} t\right)^{\frac{1}{2}}\left(\int_{t^{n-1}}^{t^{n}}\|\rho\|^{2} \mathrm{~d} t\right)^{\frac{1}{2}} .
$$

The assertion then follows by estimation of the time integral of $\|\tilde{G}\|^{2}$ :

$$
\int_{t^{n-1}}^{t^{n}}\|\tilde{G}\|^{2} \mathrm{~d} t \leq \lambda_{n}\left\|\left(I-\Pi^{n}\right) U^{n-1}\right\|^{2}+\lambda_{n}\left\|\psi_{n-1}\right\|^{2},
$$

and noting that $\psi_{n-1}=\sum_{i=1}^{n-1}\left(\Pi^{i}-\Pi^{i-1}\right) U^{i-1}$.

Lemma 5.6. With the above notation, we have

$$
\begin{aligned}
& \sum_{n=1}^{N} \int_{t^{n-1}}^{t^{n}} \frac{t-t^{n}}{\lambda_{n}}\left\langle g^{n}-g^{n-1}, \bar{v}\right\rangle \mathrm{d} t \\
& \quad \leq \sum_{n=1}^{N}\left(\lambda_{n}^{3}\left\|g^{n}-g^{n-1}\right\|^{2}+\lambda_{n}\left\|\sum_{i=1}^{n-1} \frac{\lambda_{i}}{2}\left(g^{i}-g^{i-1}\right)\right\|^{2}\right)^{\frac{1}{2}}\left(\int_{t^{n-1}}^{t^{n}}\|\rho\|^{2} \mathrm{~d} t\right)^{\frac{1}{2}} .
\end{aligned}
$$


Proof. Recalling the definition of $G$, an integration by parts with respect to time gives

$$
\sum_{n=1}^{N} \int_{t^{n-1}}^{t^{n}} \frac{t-t^{n}}{\lambda_{n}}\left\langle g^{n}-g^{n-1}, \bar{v}\right\rangle \mathrm{d} t=\sum_{n=1}^{N} \int_{t^{n-1}}^{t^{n}}\left\langle G,-\bar{v}_{t}\right\rangle \mathrm{d} t
$$

We recall the properties of $\bar{v}$ in (50) and estimate the right-hand side further:

$$
\sum_{n=1}^{N} \int_{t^{n-1}}^{t^{n}}\left\langle G,-\bar{v}_{t}\right\rangle \mathrm{d} t \leq \sum_{n=1}^{N}\left(\int_{t^{n-1}}^{t^{n}}\|G\|^{2} \mathrm{~d} t\right)^{\frac{1}{2}}\left(\int_{t^{n-1}}^{t^{n}}\|\rho\|^{2} \mathrm{~d} t\right)^{\frac{1}{2}} .
$$

The assertion then follows by estimation of the integral of $\|G\|^{2}$,

$$
\int_{t^{n-1}}^{t^{n}}\|G\|^{2} \mathrm{~d} t \leq \lambda_{n}^{3}\left\|g^{n}-g^{n-1}\right\|^{2}+\lambda_{n}\left\|\theta_{n-1}\right\|^{2}
$$

and noting that $\theta_{n-1}=\sum_{i=1}^{n-1}-\frac{\lambda_{i}}{2}\left(g^{i}-g^{i-1}\right)$.

Lemma 5.7. With the above notation, we have

$$
\sum_{n=1}^{N} \int_{t^{n-1}}^{t^{n}}\left\langle\tilde{f}^{n}-f, \bar{v}\right\rangle \mathrm{d} t \leq C_{a p p} \sum_{n=1}^{N}\left(\int_{t^{n-1}}^{t^{n}} \lambda_{n}^{2}\left\|\tilde{f}^{n}-f\right\|^{2} \mathrm{~d} t\right)^{1 / 2}\left(\int_{t^{n-1}}^{t^{n}}\|\rho\|^{2} \mathrm{~d} t\right)^{1 / 2} .
$$

Proof. As $\tilde{f}^{n}$ is the $L^{2}$ projection of $f$ in time, we have

$$
\sum_{n=1}^{N} \int_{t^{n-1}}^{t^{n}}\left\langle\tilde{f}^{n}-f, \bar{v}\right\rangle \mathrm{d} t=\sum_{n=1}^{N} \int_{t^{n-1}}^{t^{n}}\left\langle\tilde{f}^{n}-f, \bar{v}-\zeta^{n}\right\rangle \mathrm{d} t
$$

for the lowest order time approximation $\zeta^{n}(\cdot):=\lambda_{n}^{-1} \int_{t^{n-1}}^{t^{n}} \bar{v}(t, \cdot) \mathrm{d} t$ of $\bar{v}$. With the approximation property of $\zeta^{n}$ in time, we deduce

$$
\int_{t^{n-1}}^{t^{n}}\left\|\bar{v}-\zeta^{n}\right\|^{2} \mathrm{~d} t \leq C_{\text {app }}^{2} \lambda_{n}^{2} \int_{t^{n-1}}^{t^{n}}\left\|\bar{v}_{t}\right\|^{2} \mathrm{~d} t
$$

and recalling that $\bar{v}_{t}=-\rho$, the Cauchy-Schwarz inequality implies

$$
\sum_{n=1}^{N} \int_{t^{n-1}}^{t^{n}}\langle\tilde{f}-f, \bar{v}\rangle \mathrm{d} t \leq C_{\text {app }} \sum_{n=1}^{N}\left(\int_{t^{n-1}}^{t^{n}} \lambda_{n}^{2}\left\|\tilde{f}^{n}-f\right\|^{2} \mathrm{~d} t\right)^{1 / 2}\left(\int_{t^{n-1}}^{t^{n}}\|\rho\|^{2} \mathrm{~d} t\right)^{1 / 2} .
$$

To complete a posteriori error bounds in Theorem 5.2 we also need the following two lemmas in which the elliptic error terms $\epsilon$ and $\epsilon_{t}$ are estimated by fully computable residuals. 
Lemma 5.8. Let $\epsilon$ be as in (43). Then, we have

$$
\int_{0}^{T}\|\epsilon\|^{2} \mathrm{~d} t \leq \frac{2 \lambda_{N}}{3} \mathcal{E}\left(\mathcal{T}_{N}, U^{N}, g^{N}\right)+\sum_{n=1}^{N-1} \frac{4 \lambda_{n}}{3} \mathcal{E}\left(\mathcal{T}_{n}, U^{n}, g^{n}\right)^{2} .
$$

Proof. Noting that $\left(\left(t-t^{n-1}\right) / \lambda_{n}\right)^{2} \leq \frac{1}{3}$ and $\left(\left(t^{n}-t\right) \lambda_{n}\right)^{2} \leq \frac{1}{3}$, we have

$$
\int_{0}^{T}\|\epsilon\|^{2} \mathrm{~d} t \leq \sum_{n=1}^{N} \frac{2 \lambda_{n}}{3}\left(\left\|\epsilon_{n}\right\|^{2}+\left\|\epsilon_{n-1}\right\|^{2}\right)
$$

The assertion then follows by Theorem 3.2 .

Lemma 5.9. Let $\epsilon$ be as in (43) and $\tau \in[0, T]$, then, we have

$$
\int_{0}^{\tau}\left\langle\epsilon_{t}, \rho\right\rangle \mathrm{d} t \leq \sum_{n=1}^{N} \mathcal{E}\left(\hat{\mathcal{T}}_{n}, U^{n}-U^{n-1}, g^{n}-g^{n-1}\right) \max _{0 \leq t \leq T}\|\rho\|,
$$

where $\hat{\mathcal{T}}_{n}:=\mathcal{T}_{n} \cap \mathcal{T}_{n-1}$ denotes the finest common coarsening of $\mathcal{T}_{n}$ and $\mathcal{T}_{n-1}$, $n=1, \ldots, N$.

Proof. We have $\epsilon_{t}(t)=\left(\epsilon_{n}-\epsilon_{n-1}\right) / \lambda_{n}$, for $t \in\left(t_{n-1}, t_{n}\right]$ and $n=1, \ldots, N$. Denoting $\tau:=t_{r+1 / 2}$ and $r:=\max \left\{k: t_{k} \leq \tau, k=1, \ldots, N\right\}$, we then have

$$
\int_{0}^{\tau}\left\langle\epsilon_{t}, \rho\right\rangle \mathrm{d} t=\sum_{n=1}^{r+1 / 2} \int_{t^{n-1}}^{t^{n}} \frac{1}{\lambda_{n}}\left\langle\epsilon_{n}-\epsilon_{n-1}, \rho\right\rangle \mathrm{d} t \leq \max _{0 \leq t \leq T}\|\rho\| \sum_{n=1}^{r+1 / 2}\left\|\epsilon_{n}-\epsilon_{n-1}\right\| .
$$

We now observe that the finite element function $\tilde{z}$ in the proof of Theorem 3.2 can be selected from a subspace of $S^{r}$; in particular, we can select the finite element subspace corresponding to the finest common coarsening mesh $\hat{\mathcal{T}}_{n}$, for $n=1, \ldots, N$. Then, following a completely analogous argument as in the proof of of Theorem 3.2 . we can arrive to the bound

$$
\left\|\epsilon_{n}-\epsilon_{n-1}\right\| \leq \mathcal{E}\left(\hat{\mathcal{T}}_{n}, U^{n}-U^{n-1}, g^{n}-g^{n-1}\right)
$$

which already yields the result.

Remark 5.10. Note that the following simpler alternative bound for the term in Lemma 5.9 is also possible,

$$
\int_{0}^{\tau}\left\langle\epsilon_{t}, \rho\right\rangle \mathrm{d} t \leq \sum_{n=0}^{N} \mathcal{E}\left(\mathcal{T}_{n}, U^{n}, g^{n}\right) \max _{0 \leq t \leq T}\|\rho\| .
$$

This bound shifts the emphasis from the finest common coarsening mesh, $\hat{\mathcal{T}}_{n}$, in Lemma 5.9 to the elliptic estimators acting on meshes at each time-step only, which can be of practical importance when implementing adaptive algorithms based on the estimators.

Proof of Theorem 5.2. To conclude the proof, we estimate the left-hand side of (44) in each case of the test functions: $\bar{v}$ to derive $L^{2}\left(L^{2}\right)$-norm a posteriori bound and $\rho$ for the $L^{\infty}\left(L^{2}\right)$-norm bound. First we deal with the $L^{2}\left(L^{2}\right)$ case; we start by 
integrating (44) by parts in time,

$$
\begin{gathered}
\int_{0}^{T}\left\langle e_{t}, \bar{v}\right\rangle+B(\rho, \bar{v}) \mathrm{d} t=\int_{0}^{T}\left\langle e,-\bar{v}_{t}\right\rangle \mathrm{d} t+[\langle e, \bar{v}\rangle]_{0}^{T}-\int_{0}^{T} B\left(\bar{v}_{t}, \bar{v}\right) \mathrm{d} t \\
=\int_{0}^{T}\langle\rho, \rho\rangle \mathrm{d} t-\int_{0}^{T}\langle\epsilon, \rho\rangle \mathrm{d} t-\langle e(0), \bar{v}(0)\rangle-\int_{0}^{T} \frac{1}{2} \frac{d}{d t} B(\bar{v}, \bar{v}) \mathrm{d} t \\
=\int_{0}^{T}\|\rho\|^{2} \mathrm{~d} t-\int_{0}^{T}\langle\epsilon, \rho\rangle \mathrm{d} t-\langle e(0), \bar{v}(0)\rangle+\frac{1}{2} B(\bar{v}(0), \bar{v}(0)) .
\end{gathered}
$$

We also have

$$
\langle e(0), \bar{v}(0)\rangle \leq\|e(0)\|\|\bar{v}(0)\| \leq\|e(0)\| C_{\mathrm{pf}} B(\bar{v}(0), \bar{v}(0)) .
$$

Using (74) and (75) in (44) after integration over each interval $\left(t^{n-1}, t^{n}\right]$ and summation with respect to $n$, we get,

$$
\begin{aligned}
& \|\rho\|_{L^{2}\left(0, T, L^{2}(\Omega)\right)}^{2} \leq\|e(0)\|^{2} \\
& \quad+\sum_{n=1}^{N} \int_{t^{n-1}}^{t^{n}}\left(\langle\epsilon, \rho\rangle+\left\langle\left(I-\Pi^{n}\right) U_{t}, \bar{v}\right\rangle+\frac{t-t^{n}}{\lambda_{n}}\left\langle g^{n}-g^{n-1}, \bar{v}\right\rangle+\left\langle\tilde{f}^{n}-f, \bar{v}\right\rangle\right) \mathrm{d} t .
\end{aligned}
$$

The bound (40) now follows upon using the triangle inequality

$$
\|e\|_{L^{2}\left(0, T, L^{2}(\Omega)\right)} \leq\|\rho\|_{L^{2}\left(0, T, L^{2}(\Omega)\right)}+\|\epsilon\|_{L^{2}\left(0, T, L^{2}(\Omega)\right)},
$$

Young's inequality and Lemmas 5.5, 5.6, 5.7 and 5.8.

For the $L^{\infty}\left(L^{2}\right)$-norm case, upon testing with $v=\rho$, we deduce for the left-hand side of (44) by integrating by parts to some $\tau \in[0, T]$,

$$
\int_{0}^{\tau}\left\langle e_{t}, \rho\right\rangle+B(\rho, \rho) \mathrm{d} t=\|\rho(\tau)\|^{2}-\|\rho(0)\|^{2}-\int_{0}^{\tau}\left\langle\epsilon_{t}, \rho\right\rangle \mathrm{d} t+\int_{0}^{\tau} B(\rho, \rho) \mathrm{d} t .
$$

Choosing $\tau$ such that $\|\rho(\tau)\|=\max _{0 \leq t \leq T}\|\rho\|$, using the triangle inequality, $\|\rho(0)\| \leq$ $\|e(0)\|+\|\epsilon(0)\|$, and (77) in (44), we get,

$$
\begin{aligned}
& \|\rho\|_{L^{\infty}\left(0, T, L^{2}(\Omega)\right)}^{2}+\int_{0}^{\tau} B(\rho, \rho) \mathrm{d} t \\
& \quad \leq\|e(0)\|^{2}+\|\epsilon(0)\|^{2}+\int_{0}^{\tau}\left(\left\langle\epsilon_{t}, \rho\right\rangle+\left\langle\tilde{G}_{t}, \rho\right\rangle+\left\langle G_{t}, \rho\right\rangle+\left\langle\tilde{f}^{n}-f, \rho\right\rangle\right) \mathrm{d} t
\end{aligned}
$$

where $G$ and $\tilde{G}$ are given by (52) and (51). The bound (39) follows again using triangle inequality

$$
\|e\|_{L^{\infty}\left(0, T, L^{2}(\Omega)\right)} \leq\|\rho\|_{L^{\infty}\left(0, T, L^{2}(\Omega)\right)}+\|\epsilon\|_{L^{\infty}\left(0, T, L^{2}(\Omega)\right)},
$$

Lemmas 5.4 and 5.9 as well as $\max _{0 \leq t \leq T}\|\epsilon\| \leq \max _{0 \leq t \leq T} \mathcal{E}\left(\mathcal{T}_{n}, U^{n}, g^{n}\right)$. 
We note that a posteriori bounds in the $L^{2}\left(H^{2}\right)$-norm of the error have been already considered in 43 , along with their application within an adaptive algorithm. The $L^{2}\left(H^{2}\right)$-norm theoretical and numerical results appear to be of the expected order of convergence; they are omitted here for brevity.

\section{NUMERICAL EXPERIMENTS}

For $t \in[0,1]$ and $\Omega:=(0,1)^{2}$, we consider two benchmark problems for which $u_{0}$ and $f$ are chosen so that the exact solution $u$ of problem (31) coincides with one of the following solutions:

$$
\begin{gathered}
u_{1}(x, y, t)=\sin (\pi t) 10^{2} \sin ^{2}(\pi x) \sin ^{2}(\pi y) e^{-10\left(x^{2}+y^{2}\right)}, \\
u_{2}(x, y, t)=\sin (20 \pi t) \sin ^{2}(\pi x) \sin ^{2}(\pi y) e^{-10\left(x^{2}+y^{2}\right)} .
\end{gathered}
$$

Solutions $u_{1}$ and $u_{2}$ are both smooth but $u_{2}$ oscillates much faster where as $u_{1}$ exhibits greater space dependency of the error. They are defined so as to emphasize different aspects of the estimators at hand. Similar examples have been studied elsewhere, for example in 43 ] in the context of $L^{2}\left(H^{2}\right)$-norm a posteriori estimators; see also 29, 33, 34 for similar examples in the context of second order problems.

For the numerical experiments, the library FEniCS was used; see (http://fenicsproject.org/). For each of the examples, we compute the solution of (32) using quadratic polynomials, conforming meshes consisting of triangular elements and interior penalty parameters $\sigma_{0}=\xi_{0}=20$ in (18), which are sufficient to guarantee stability of the numerical scheme. The interior penalty parameters have a known effect on the effectivity indices; cf., [32,43]. Note that in Figures 18 the $x$-axis depicts the time domain of the problem, i.e., the interval $[0,1]$.

We study the asymptotic behavior of the indicators by setting all constants appearing in Theorem 5.2 equal to one. We monitor the evolution of the values along the time domain of the problem and the experimental order of convergence of the estimators and the error as well as of the effectivity index over time on a sequence of uniformly refined meshes with $h_{\kappa, i}:=2^{-i / 2-1}, i=1, \ldots, 6, \kappa \in \mathcal{T}$ with fixed time-steps $\lambda \approx \max _{\kappa} h_{\kappa}^{3}$ and $\lambda \approx \max _{\kappa} h_{\kappa}^{2}$. To this end, we define experimental order of convergence $(E O C)$ of a given sequence of positive quantities $a(i)$ defined on a sequence of meshes of size $h(i)$ by

$$
\operatorname{EOC}(a, i)=\frac{\log (a(i+1) / a(i))}{\log (h(i+1) / h(i))}
$$

the accumulated coarsening or mesh change estimators by

$$
\mathbb{E}_{\text {coarsen }, \infty, m}:=\left(\sum_{n=1}^{m} \gamma_{\infty, n} \lambda_{n}\right)^{\frac{1}{2}} \quad \text { and } \quad \mathbb{E}_{\text {coarsen }, 2, m}:=\left(\sum_{n=1}^{m} \gamma_{2, n} \lambda_{n}\right)^{\frac{1}{2}},
$$

the accumulated time error evolution estimators by

$$
\begin{aligned}
\mathbb{E}_{\mathrm{time}, \infty, m} & :=\left(\sum_{n=1}^{m}\left(\eta_{\infty, n}+\beta_{\infty, n}\right) \lambda_{n}+\sum_{n=1}^{m} \tilde{\eta}_{\infty, n}\right)^{\frac{1}{2}} \text { and } \\
\mathbb{E}_{\mathrm{time}, 2, m} & :=\left(\sum_{n=1}^{m}\left(\eta_{2, n}+\beta_{2, n}\right) \lambda_{n}\right)^{\frac{1}{2}}
\end{aligned}
$$


the accumulated space error estimators by

$$
\begin{aligned}
\mathbb{E}_{\text {space }, \infty, m} & :=\max _{0 \leq n \leq N}\left\{\mathcal{E}\left(\mathcal{T}_{n}, U^{n}, g^{n}\right)\right\} \quad \text { and } \\
\mathbb{E}_{\text {space, }, m}: & :=\left(\sum_{n=1}^{m} \mathcal{E}\left(\mathcal{T}_{n}, U^{n}, g^{n}\right)^{2} \lambda_{n}\right)^{\frac{1}{2}}
\end{aligned}
$$

and the inverse effectivity index

$$
I E I_{m}=\frac{\|e\|_{L^{\infty}\left(0, t_{m} ; L^{2}(\Omega)\right)}}{\mathbb{E}_{\mathrm{time}, \infty, m}+\mathbb{E}_{\mathrm{space}, \infty, m}} \quad \text { or } \quad I E I_{m}=\frac{\|e\|_{L^{2}\left(0, t_{m} ; L^{2}(\Omega)\right)}}{\mathbb{E}_{\mathrm{time}, 2, m}+\mathbb{E}_{\mathrm{space}, 2, m}},
$$

for the cases $L^{\infty}\left(L^{2}\right)$ - and $L^{2}\left(L^{2}\right)$-error, respectively. The IEI conveys the same information as the (standard) effectivity index and has the advantage of relating directly to the constants appearing in Theorem 5.2

The results of numerical experiments on uniform meshes, depicted in Figures 1 4. indicate that the error estimators are reliable and also efficient which can be seen from the effectivity index behaviour and the EOC of the error and the time and space estimators for both $L^{2}\left(L^{2}\right)$ - and $L^{\infty}\left(L^{2}\right)$-norm a posteriori bounds.

To further evaluate practical aspects of the derived a posteriori estimators, they are incorporated within two adaptive algorithms; these are outlined in pseudocode as follows:
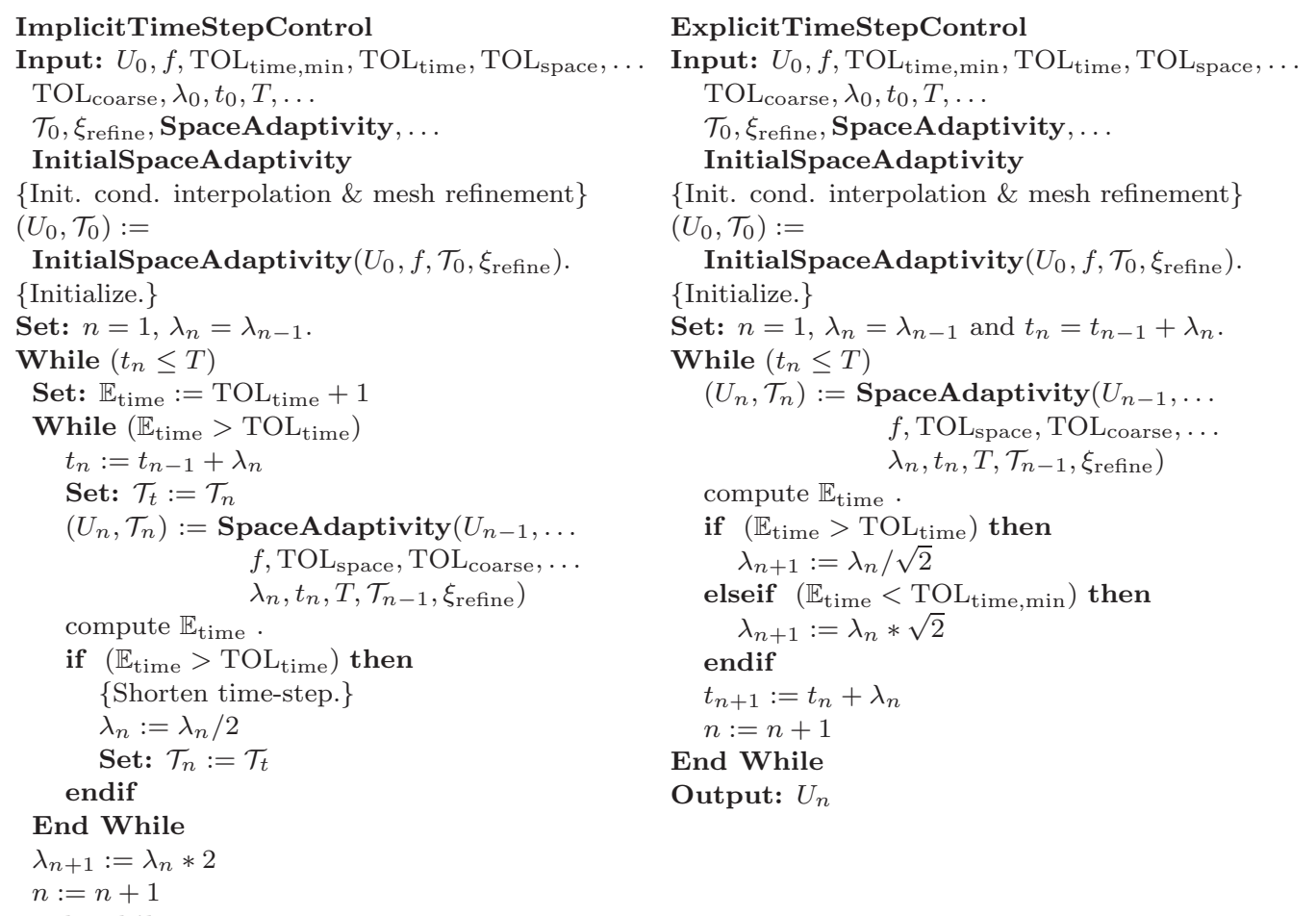

End While

Output: $U_{n}$

End While

Output: $U_{n}$

where SpaceAdaptivity (and InitialSpaceAdaptivity) are performed using a standard Dörfler marking strategy expressed in pseudocode as follows: 


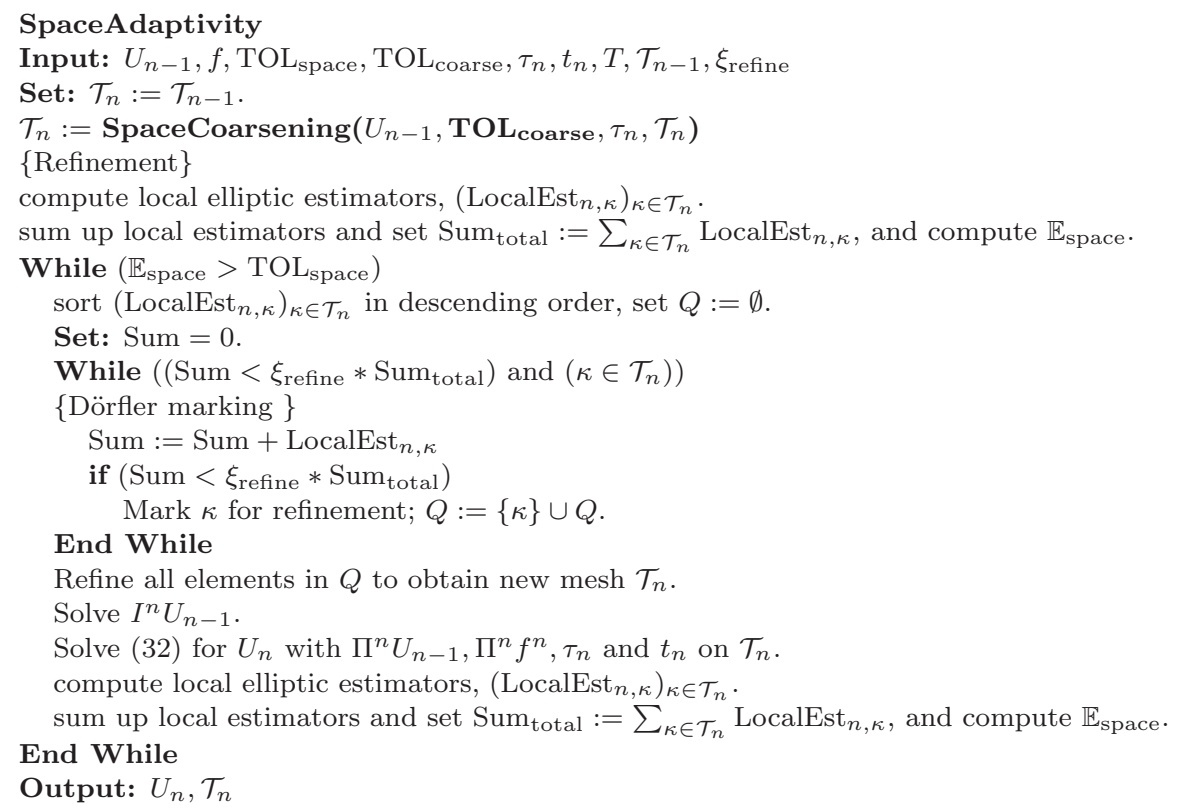

The refinement ratio $0<\xi_{\text {refine }} \leq 1$ and the tolerances $\mathrm{TOL}_{\text {space }}>0, \mathrm{TOL}_{\text {space }}>0$ and $\mathrm{TOL}_{\text {coarse }}>0$ are predefined quantities. The value of $\xi_{\text {refine }}:=0.75$ was used throughout the experiments in adaptive algorithms. Note that the coarsening tolerance, $\mathrm{TOL}_{\text {coarse, }}$ (as well as the tolerance for the alternative space estimator in $L^{\infty}$ case of Remark 5.10 had to be determined experimentally for given space and time tolerances and depending on an example.

In summary, time-step and mesh adaptivity are performed separately in both algorithms described above. More specifically, in the explicit variant of the algorithm, the time-step search is performed by only shortening the time-step if the time estimators are above the predefined tolerance and otherwise increasing the time-step by a constant factor. By doing this, one avoids the costly effort of the time-step search which would enforce all estimators under a certain tolerance but, on the other hand, convergence to a set tolerance is no longer guaranteed. In the implicit variant of the adaptivity the time-steps are searched to suit the condition that all estimators can be guaranteed to reduce under predefined tolerances. Terminology used here is somewhat analog to the explicit (i.e., marching) and implicit integration methods used to solve time dependent problems.

The results of experiments with adaptive algorithms as well as a comparison between the two algorithms, are detailed in Figures 5,8 where we monitor timestep size, accumulated degrees of freedom and error evolution in comparison to the uniform approach leading to the desired tolerance. The accumulated degrees of freedom are given by

$$
\text { acc. DOFs }:=\sum_{n=1}^{N} \operatorname{dim}\left(S^{r, n}\right) .
$$

The results of these test cases imply substantial reduction in degrees of freedom by both adaptive algorithms in order to reach the same error tolerance as compared 
with the uniform approach. This implies a potential efficiency gain in solving PDE problems addressed in this work.

The estimators presented above are found to be suitable for both adaptive timestepping algorithms due to their good separated scaling properties in time and in space. The numerical results appear to be less sensitive to mesh change, compared to the same adaptive algorithms based on the $L^{2}\left(H^{2}\right)$-norm a posteriori error estimators presented in [43]. For instance, terms involving $\left(g^{n}-g^{n-1}\right)$ which is sensitive to mesh change (coarsening as well as refinement) scale down sufficiently fast with the a posteriori estimators presented in this work, resulting to robust error reduction in an adaptive algorithm.

It is also perhaps worth noting that if one sums up the overall degrees of freedom resulting from all solution cycles of one of the adaptive algorithms and thus including the search steps for suitable time-step as well as mesh refinement, then the presented implementations of the adaptive algorithms reduce the errors with less degrees of freedom than is required in the uniform approach. This can be seen in the results concerning both adaptive algorithms. Indeed, if one was to overestimate that an average time-step search and corresponding mesh refinement steps would require, say, ten times as many degrees of freedom as the accumulated degrees of freedom, then the presented adaptive algorithms would still lead to at least the same error reduction as the uniform approach but with fewer degrees of freedom. However, this error reduction property of an adaptive algorithm depends greatly on the chosen tolerances, refinement ratios as well as the coefficient for time-step search steering (here the values $\sqrt{2}$ and $1 / \sqrt{2}$ and 2 and $1 / 2$ were used for this purpose in explicit an implicit algorithms respectively).

Finally, we note that the considerably more computationally efficient ExplicitTimeStepControl algorithm (due to absence of time-step searching step) was found to reach desired error tolerances (even though this is not guaranteed, in general) in these numerical experiments.

\section{Concluding Remarks}

Residual type a posteriori estimates of errors measured in $L^{\infty}\left(L^{2}\right)$ and $L^{2}\left(L^{2}\right)$ norms for a numerical scheme consisting of implicit Euler method in time and discontinuous Galerkin method of local polynomial degrees $r \geq 2$ in space for linear parabolic fourth order problems in space dimensions 2 and 3 are presented. Numerical experiments confirming the practical efficiency and reliability of the a posteriori estimators are also presented, along with the use of these a posteriori estimator within adaptive algorithms. It appears that the derived a posteriori bounds and the respective adaptive algorithms can be modified in a straightforward fashion to the original $\mathrm{dG}$ method of Baker [3] and to the $C^{0}$-interior penalty methods of [9, 17, as well as the incorporation of different types of a posteriori bounds for the "elliptic" part of the error [38. We also believe that it is possible to easily extend this approach to derive a posteriori bounds for classical non-conforming spatial discretizations for fourth order problems [5, 12. Moreover, second order operators can be included in the present analysis, as was done in [43. An extension of these results to non-linear fourth order parabolic equations remains a future challenge. 

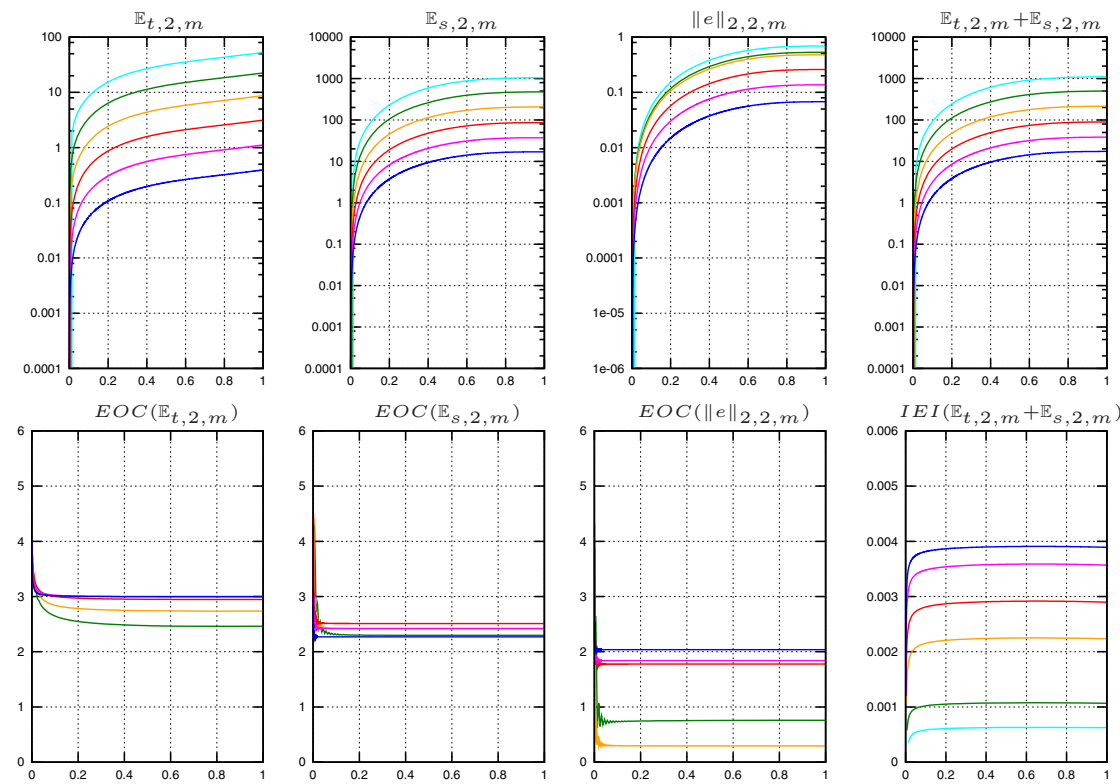

(a) Example 80 solution with fixed time-step $\lambda \approx h^{2}$. Note the asymptotic trend of the inverse effectivity index towards value 0.004 with respect to the finest mesh, $h_{\kappa, 6}$ and the corresponding time-step.
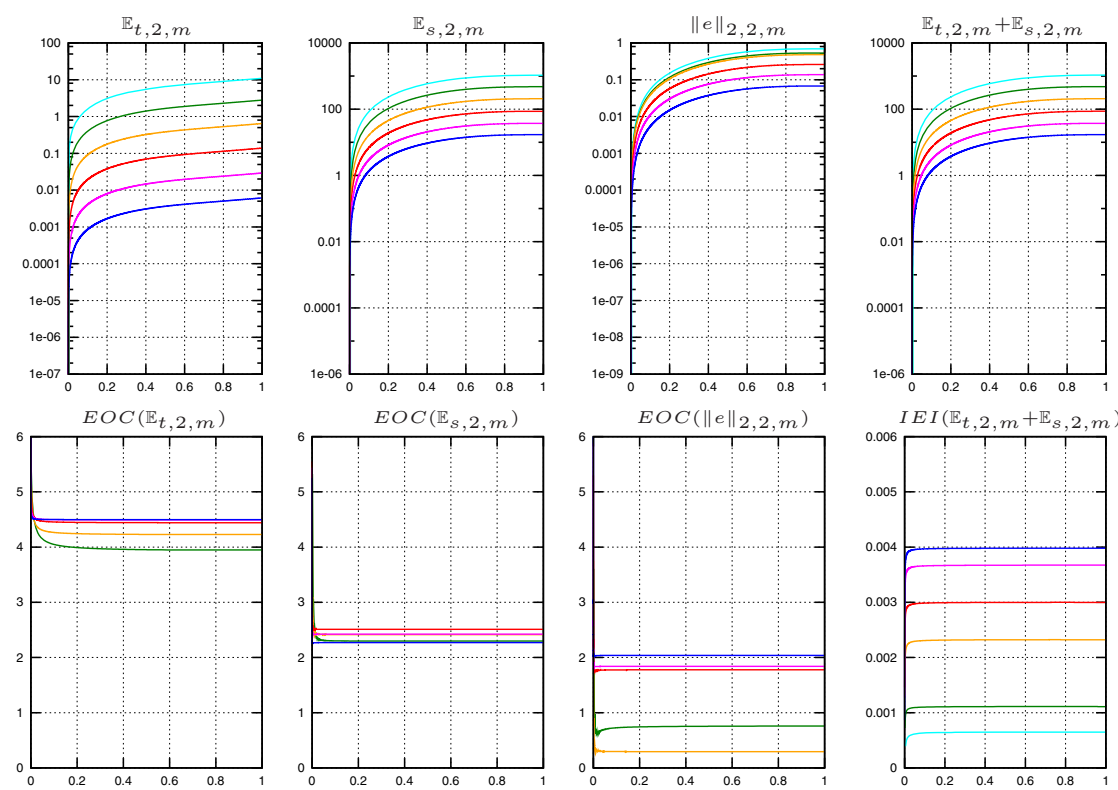

(b) Example 80 solution with fixed time-step $\lambda \approx h^{3}$. Note the asymptotic trend of the inverse effectivity index towards value 0.004 with respect to the finest mesh, $h_{\kappa, 6}$ and the corresponding time-step.

Figure 1. Example (80) with solution $u_{1}, L^{2}$ estimators and error. The $\mathrm{x}$-axis depicts the time domain of the problem, i.e., $t \in[0,1]$ and the colors indicate mesh level $h_{\kappa, i}:=2^{-i / 2-1}, i=1, \ldots, 6, \kappa \in \mathcal{T}$, i.e., the blue lines are obtained with the finest mesh $h_{\kappa, 6}$. 

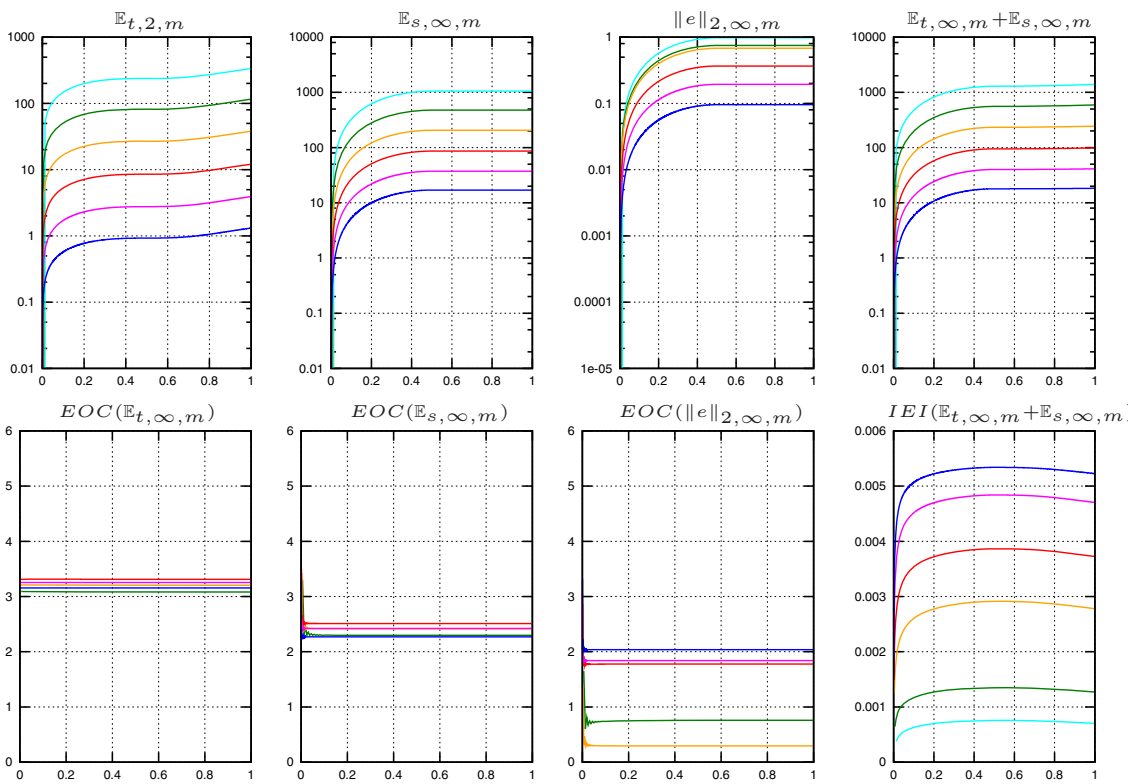

(a) Example 80 solution with fixed time-step $\lambda \approx h^{2}$. Note the asymptotic trend of the inverse effectivity index towards value between 0.005 and 0.006 with respect to the finest mesh, $h_{\kappa, 6}$ and the corresponding time-step.
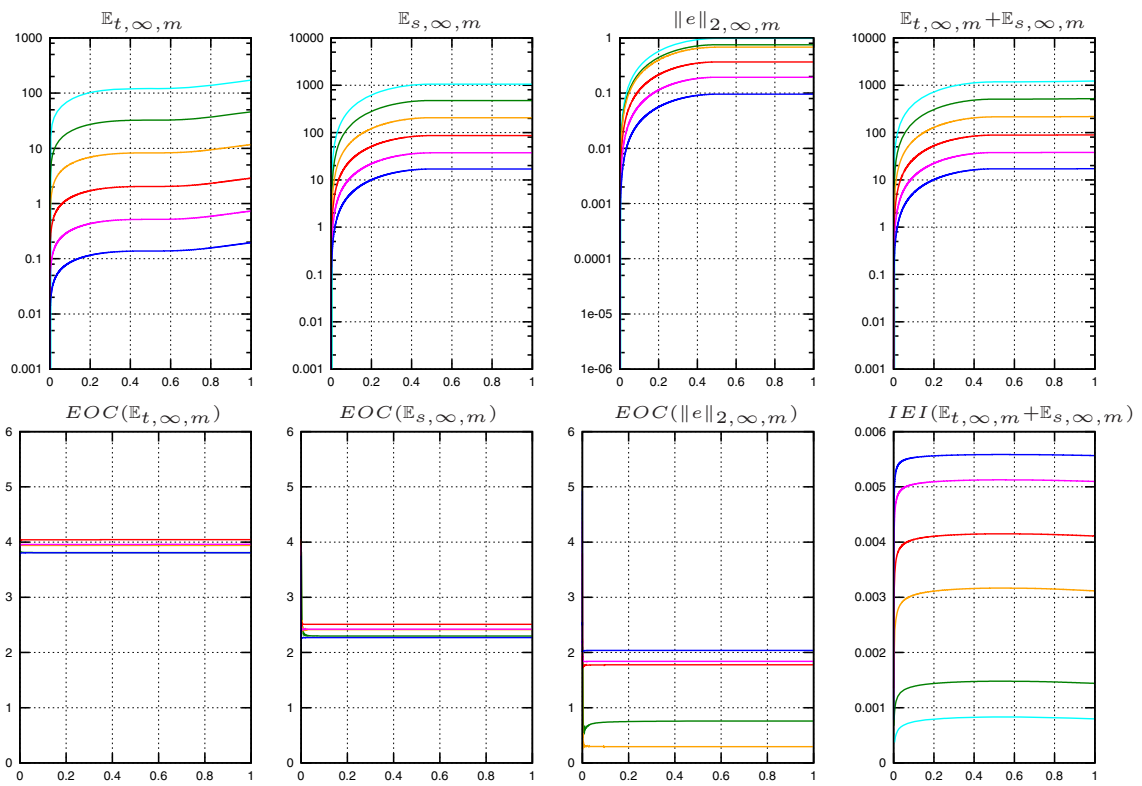

(b) Example 80 solution with fixed time-step $\lambda \approx h^{3}$. Note the asymptotic trend of the inverse effectivity index towards towards value between 0.005 and 0.006 with respect to the finest mesh, $h_{\kappa, 6}$ and the corresponding time-step.

Figure 2. Example (80) with solution $u_{1}, L^{\infty}$ estimators and error. The $\mathrm{x}$-axis depicts the time domain of the problem, i.e., $t \in[0,1]$ and the colors indicate mesh level $h_{\kappa, i}:=2^{-i / 2-1}, i=1, \ldots, 6, \kappa \in \mathcal{T}$, i.e., the blue lines are obtained with the finest mesh $h_{\kappa, 6}$. 

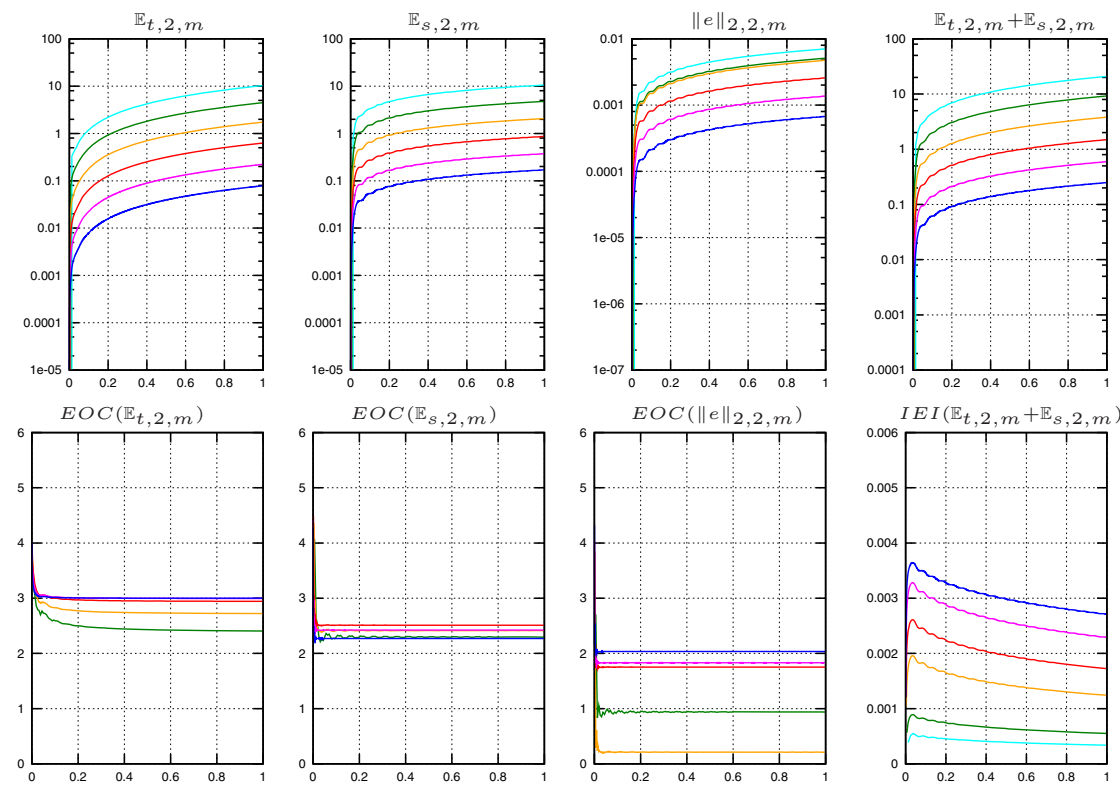

(a) Example 81] solution with fixed time-step $\lambda \approx h^{2}$. Note the asymptotic trend of the inverse effectivity index towards value 0.004 with respect to the finest mesh, $h_{\kappa, 6}$ and the corresponding time-step at the start of the interval. The IEI then drops as the time estimator starts to emphasize the $I E I$ behaviour as the time dependency of the error in this problem is dominating.

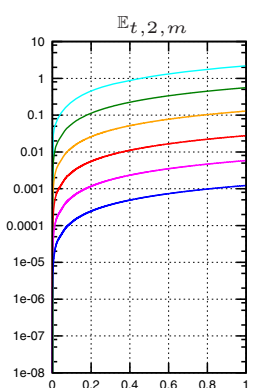

$\operatorname{EOC}\left(\mathbb{E}_{t, 2, m}\right)$



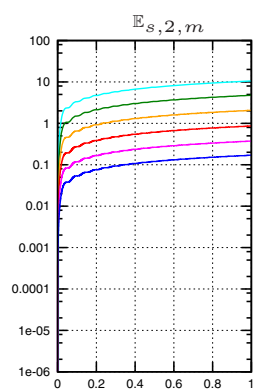

$\operatorname{EOC}\left(\mathbb{E}_{s, 2, m}\right)$

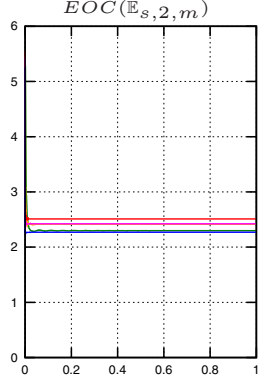

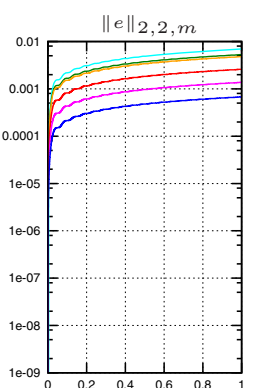

$E O C\left(\|e\|_{2}, 2, m\right)$

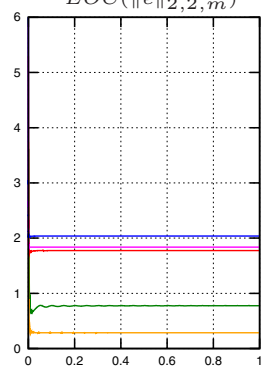

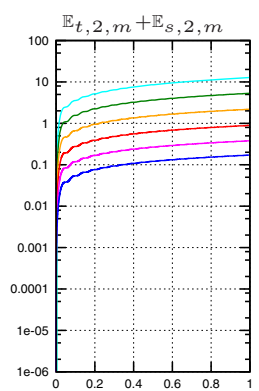

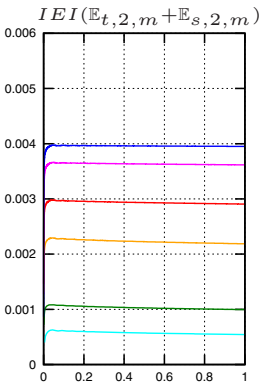

(b) Example 81 solution with fixed time-step $\lambda \approx h^{3}$. Note the asymptotic trend of the inverse effectivity index towards value 0.004 with respect to the finest mesh, $h_{\kappa, 6}$ and the corresponding time-step.

Figure 3. Example (81) with solution $u_{2}, L^{2}$ estimators and error. The xaxis depicts the time domain of the problem, i.e., $t \in[0,1]$ and the colors indicate mesh level $h_{\kappa, i}:=2^{-i / 2-1}, i=1, \ldots, 6, \kappa \in \mathcal{T}$, i.e., the blue lines are obtained with the finest mesh $h_{\kappa, 6}$. 

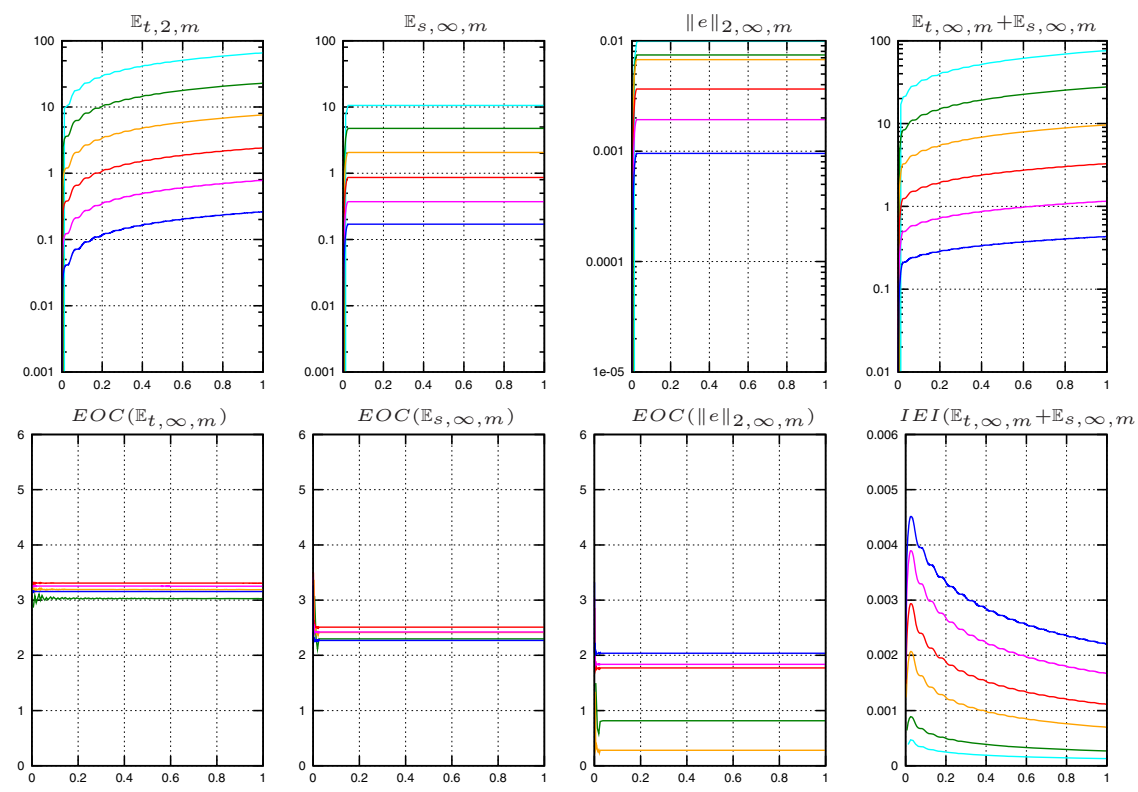

(a) Example s1 solution with fixed time-step $\lambda \approx h^{2}$. Notice the behaviour of the inverse effectivity index towards value between 0.004 and 0.005 with respect to the finest mesh, $h_{\kappa, 6}$ and the corresponding time-step at the start of the interval. The $I E I$ then drops sharply as the time estimator starts to emphasize the IEI behaviour as the time dependency of the error in this problem is dominating.
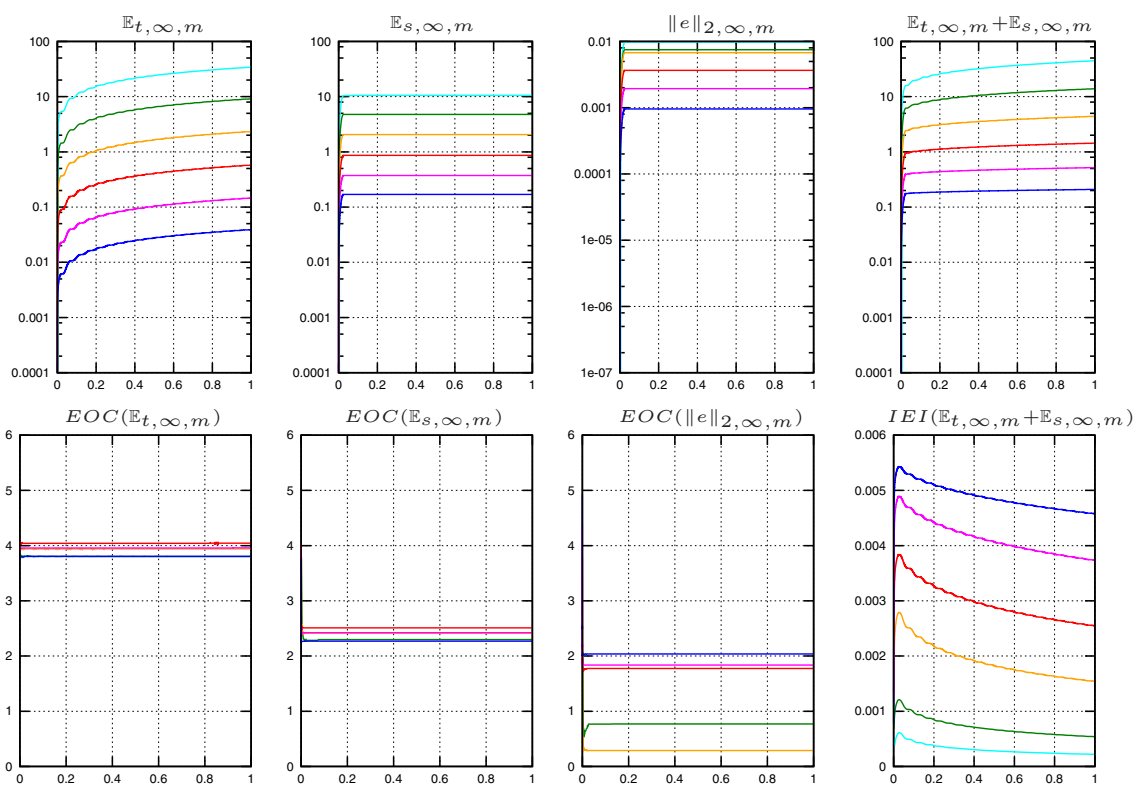

(b) Example 81 solution with fixed time-step $\lambda \approx h^{3}$. Note the asymptotic trend of the inverse effectivity index towards value between 0.005 and 0.006 with respect to the finest mesh, $h_{\kappa, 6}$ and the corresponding time-step. Also, note the significant improvement on the IEI behaviour as the time-step is refined.

FiguRE 4. Example (81) with solution $u_{2}, L^{\infty}$ estimators and error. The x-axis depicts the time domain of the problem, i.e., $t \in[0,1]$ and the colors indicate mesh level $h_{\kappa, i}:=2^{-i / 2-1}, i=1, \ldots, 6, \kappa \in \mathcal{T}$, ie. the blue lines are obtained with the finest mesh $h_{\kappa, 6}$. 

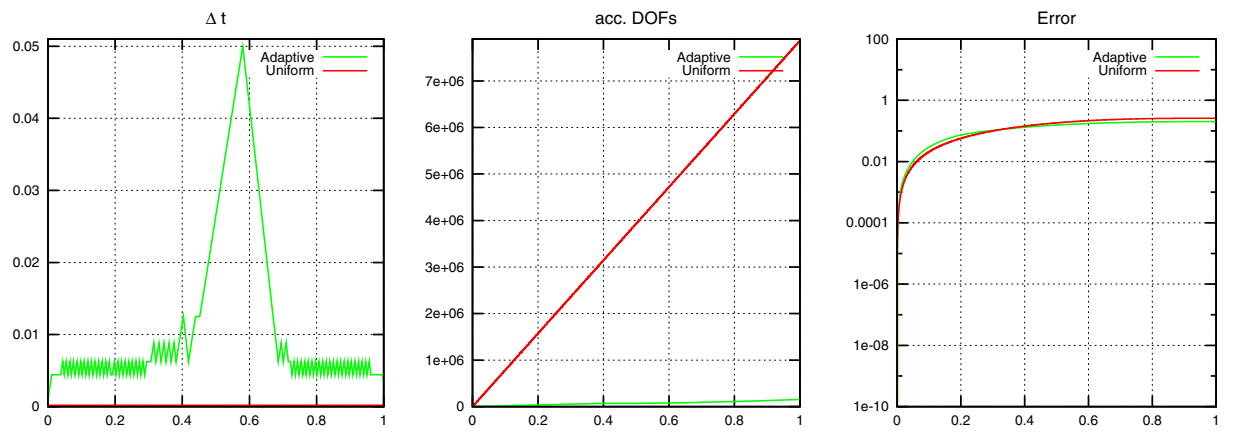

(a) ExplicitTimeStepControl algorithm in comparison to the uniform approach converging to the $L^{2}$ error tolerance $\approx 0.26$. Depicted from the left are: time-step length, accumulated degrees of freedom and error evolution over time.
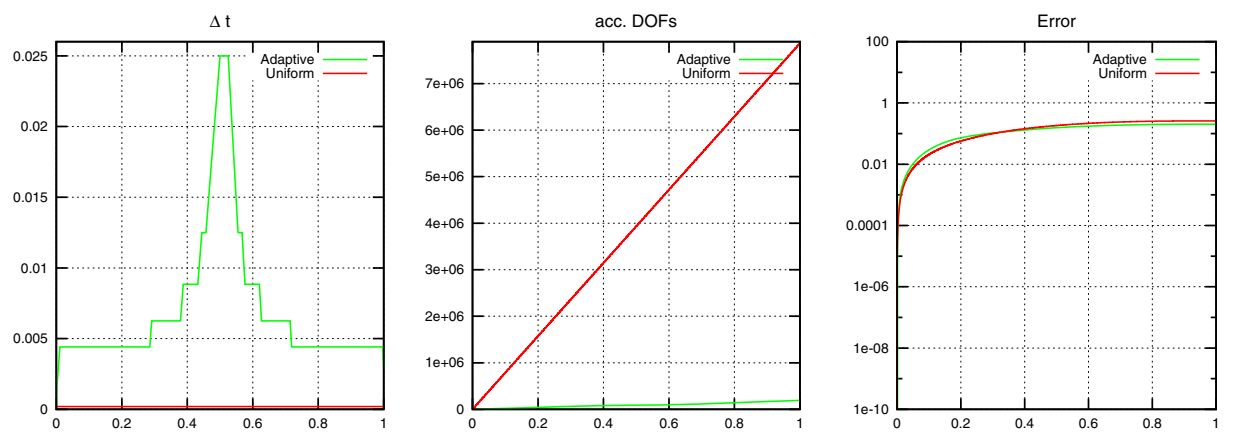

(b) ImplicitTimeStepControl algorithm in comparison to the uniform approach converging to the $L^{2}$ error tolerance $\approx 0.26$. Depicted from the left are: time-step length, accumulated degrees of freedom and error evolution over time.

Figure 5. Example (80) with solution $u_{1}$, adaptive algorithms based on $L^{2}$ estimators. The x-axis depicts the time domain of the problem, i.e., $t \in[0,1]$, the green line denotes the observed quantity obtained by the adaptive algorithm and the red line denotes the observed quantity obtained by the uniform approach. 

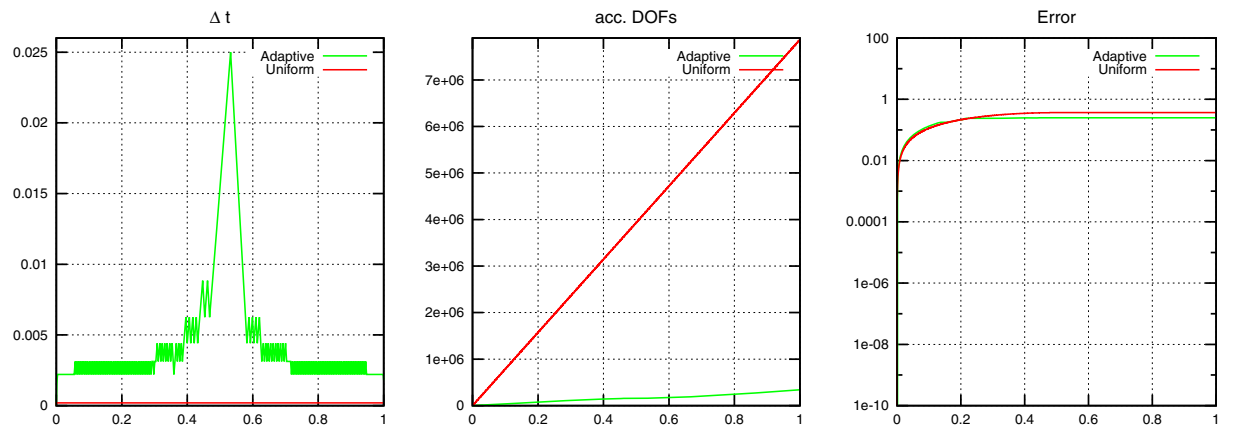

(a) ExplicitTimeStepControl algorithm in comparison to the uniform approach converging to the $L^{\infty}$ error tolerance $\approx 0.37$. Depicted from the left are: time-step length, accumulated degrees of freedom and error evolution over time.
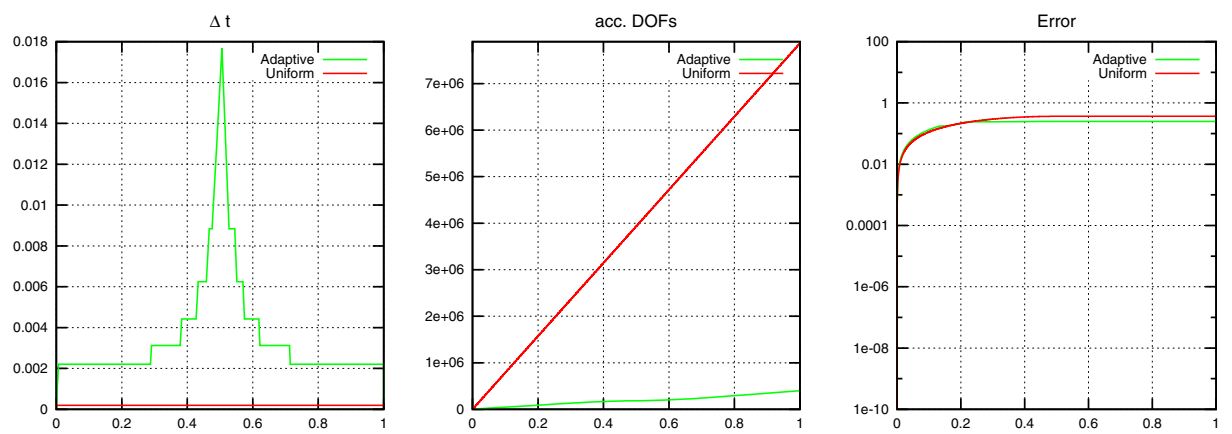

(b) ImplicitTimeStepControl algorithm in comparison to the uniform approach converging to the $L^{\infty}$ error tolerance $\approx 0.37$. Depicted from the left are: time-step length, accumulated degrees of freedom and error evolution over time.

Figure 6. Example (80) with solution $u_{1}$, adaptive algorithms based on $L^{\infty}$ estimators. The x-axis depicts the time domain of the problem, i.e., $t \in[0,1]$, the green line denotes the observed quantity obtained by the adaptive algorithm and the red line denotes the observed quantity obtained by the uniform approach. 

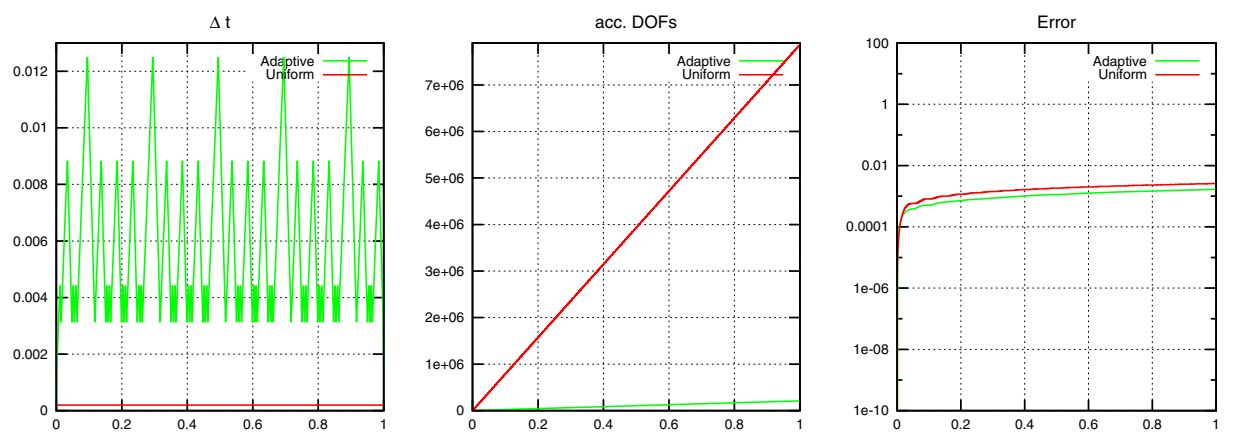

(a) ExplicitTimeStepControl algorithm in comparison to the uniform approach converging to the $L^{2}$ error tolerance $\approx 0.0026$. Depicted from the left are: time-step length, accumulated degrees of freedom and error evolution over time.
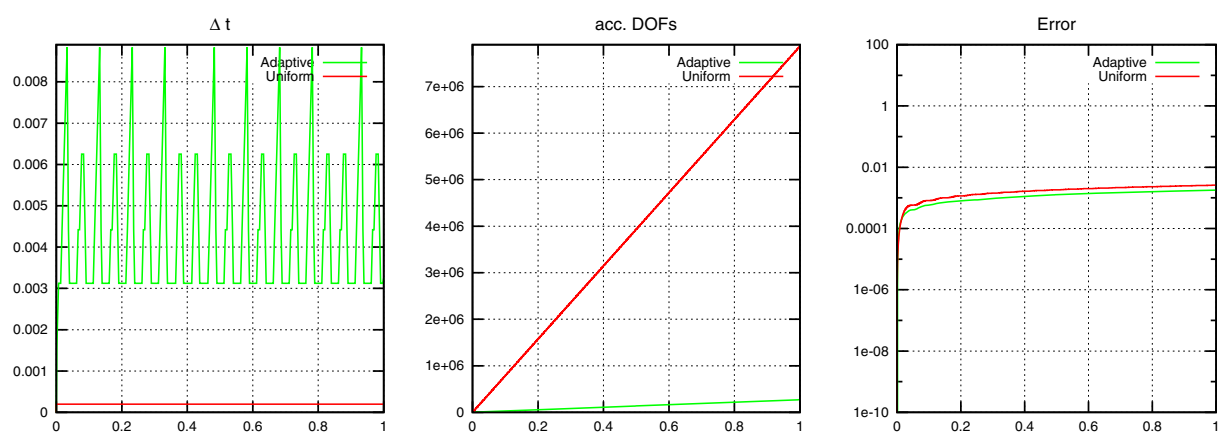

(b) ImplicitTimeStepControl algorithm in comparison to the uniform approach converging to the $L^{2}$ error tolerance $\approx 0.0026$. Depicted from the left are: time-step length, accumulated degrees of freedom and error evolution over time.

Figure 7. Example (81) with solution $u_{2}$, adaptive algorithms based on $L^{2}$ estimators. The x-axis depicts the time domain of the problem, i.e., $t \in[0,1]$, the green line denotes the observed quantity obtained by the adaptive algorithm and the red line denotes the observed quantity obtained by the uniform approach. 

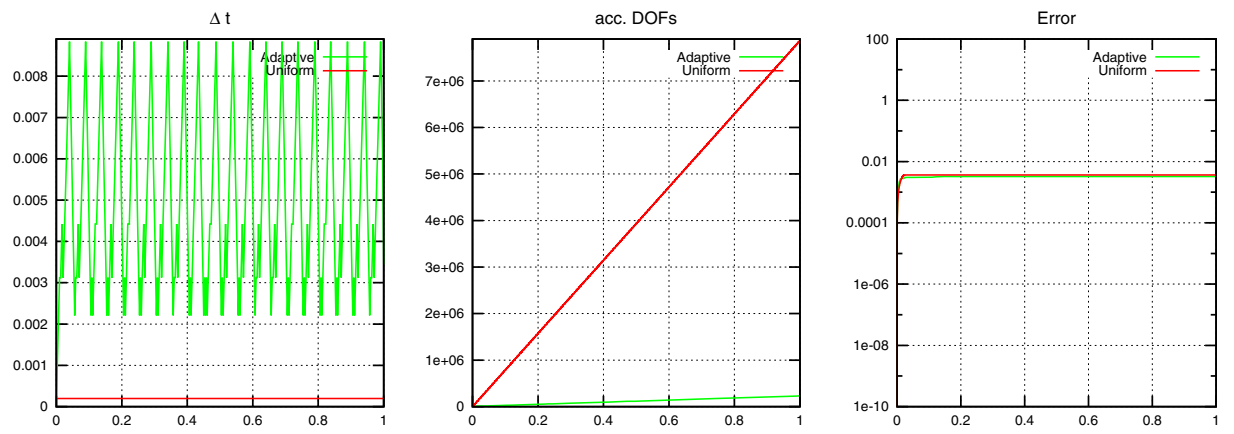

(a) ExplicitTimeStepControl algorithm in comparison to the uniform approach converging to the $L^{\infty}$ error tolerance $\approx 0.0037$. Depicted from the left are: time-step length, accumulated degrees of freedom and error evolution over time.


(b) ImplicitTimeStepControl algorithm in comparison to the uniform approach converging to the $L^{\infty}$ error tolerance $\approx 0.0037$. Depicted from the left are: time-step length, accumulated degrees of freedom and error evolution over time.

FIgURE 8. Example (81) with solution $u_{2}$, adaptive algorithms based on $L^{\infty}$ estimators. The x-axis depicts the time domain of the problem, i.e., $t \in[0,1]$, the green line denotes the observed quantity obtained by the adaptive algorithm and the red line denotes the observed quantity obtained by the uniform approach.

\section{REFERENCES}

[1] S. Adjerid, A posteriori error estimates for fourth-order elliptic problems, Comput. Methods Appl. Mech. Engrg. 191 (2002), no. 23-24, 2539-2559, DOI 10.1016/S0045-7825(01)00412-1. MR.1902705 (2003j:65105)

[2] G. A. Baker, Error estimates for finite element methods for second order hyperbolic equations, SIAM J. Numer. Anal. 13 (1976), no. 4, 564-576. MR0423836 (54:11810)

[3] G. A. Baker, Finite element methods for elliptic equations using nonconforming elements, Math. Comp. 31 (1977), no. 137, 45-59. MR0431742 (55:4737)

[4] Ľ. Ban̆as and R. Nürnberg, A posteriori estimates for the Cahn-Hilliard equation with obstacle free energy, M2AN Math. Model. Numer. Anal. 43 (2009), no. 5, 1003-1026, DOI 10.1051/m2an/2009015. MR2559742(2010i:65183)

[5] L. Beirão da Veiga, J. Niiranen, and R. Stenberg, A posteriori error estimates for the Morley plate bending element, Numer. Math. 106 (2007), no. 2, 165-179, DOI 10.1007/s00211-0070066-1. MR2291934 (2008b:74065)

[6] D. Braess, Finite Elements: Theory, Fast Solvers, and Applications in Solid Mechanics, 2nd ed., Cambridge University Press, Cambridge, 2001. MR.1827293 (2001k:65002)

[7] S. C. Brenner, T. Gudi, and L.-y. Sung, An a posteriori error estimator for a quadratic $C^{0}$. interior penalty method for the biharmonic problem, IMA J. Numer. Anal. 30 (2010), no. 3, 777-798, DOI 10.1093/imanum/drn057. MR2670114 (2011m:65268) 
[8] S. C. Brenner and L. R. Scott, The Mathematical Theory of Finite Element Methods, 2nd ed., Texts in Applied Mathematics, vol. 15, Springer-Verlag, New York, 2002. MR 1894376 (2003a:65103)

[9] S. C. Brenner and L.-Y. Sung, $C^{0}$ interior penalty methods for fourth order elliptic boundary value problems on polygonal domains, J. Sci. Comput. 22/23 (2005), 83-118, DOI 10.1007/s10915-004-4135-7. MR2142191 (2005m:65258)

[10] S. C. Brenner, K. Wang, and J. Zhao, Poincaré-Friedrichs inequalities for piecewise $H^{2}$ functions, Numer. Funct. Anal. Optim. 25 (2004), no. 5-6, 463-478, DOI 10.1081/NFA200042165. MR2106270(2005i:65178)

[11] F. Brezzi and M. Fortin, Mixed and Hybrid Finite Element Methods, Springer Series in Computational Mathematics, vol. 15, Springer-Verlag, New York, 1991. MR1115205(92d:65187)

[12] C. Carstensen and J. Hu, A posteriori error analysis for conforming MITC elements for Reissner-Mindlin plates, Math. Comp. 77 (2008), no. 262, 611-632, DOI 10.1090/S00255718-07-02028-5. MR2373172(2009a:74112)

[13] A. Charbonneau, K. Dossou, and R. Pierre, A residual-based a posteriori error estimator for the Ciarlet-Raviart formulation of the first biharmonic problem, Numer. Methods Partial Differential Equations 13 (1997), no. 1, 93-111, DOI 10.1002/(SICI)10982426(199701)13:1〈93::AID-NUM7〉3.3.CO;2-G. MR.1426319 (97m:65197)

[14] P. G. Ciarlet, The Finite Element Method for Elliptic Problems, Classics in Applied Mathematics, vol. 40, Society for Industrial and Applied Mathematics (SIAM), Philadelphia, PA, 2002. Reprint of the 1978 original [North-Holland, Amsterdam; MR0520174 (58 \#25001)]. MR.1930132

[15] P. Destuynder and M. Salaun, Mathematical Analysis of Thin Plate Models (English, with French summary), Mathématiques \& Applications (Berlin) [Mathematics \& Applications], vol. 24, Springer-Verlag, Berlin, 1996. MR1422248(2001e:74056)

[16] T. Dupont and R. Scott, Polynomial approximation of functions in Sobolev spaces, Math. Comp. 34 (1980), no. 150, 441-463, DOI 10.2307/2006095. MR559195(81h:65014)

[17] G. Engel, K. Garikipati, T. J. R. Hughes, M. G. Larson, L. Mazzei, and R. L. Taylor, Continuous/discontinuous finite element approximations of fourth-order elliptic problems in structural and continuum mechanics with applications to thin beams and plates, and strain gradient elasticity, Comput. Methods Appl. Mech. Engrg. 191 (2002), no. 34, 3669-3750, DOI 10.1016/S0045-7825(02)00286-4. MR1915664 (2003d:74086)

[18] K. Eriksson and C. Johnson, Adaptive finite element methods for parabolic problems. I. A linear model problem, SIAM J. Numer. Anal. 28 (1991), no. 1, 43-77, DOI 10.1137/0728003. MR:1083324(91m:65274)

[19] K. Eriksson and C. Johnson, Adaptive finite element methods for parabolic problems. II. Optimal error estimates in $L_{\infty} L_{2}$ and $L_{\infty} L_{\infty}$, SIAM J. Numer. Anal. 32 (1995), no. 3, 706-740, DOI 10.1137/0732033. MR1335652 (96c:65162)

[20] K. Eriksson and C. Johnson, Adaptive finite element methods for parabolic problems. IV. Nonlinear problems, SIAM J. Numer. Anal. 32 (1995), no. 6, 1729-1749, DOI 10.1137/0732078. MR.1360457(96i:65081)

[21] K. Eriksson and C. Johnson, Adaptive finite element methods for parabolic problems. $V$. Longtime integration, SIAM J. Numer. Anal. 32 (1995), no. 6, 1750-1763, DOI 10.1137/0732079. MR.1360458 (96i:65082)

[22] K. Eriksson, C. Johnson, and S. Larsson, Adaptive finite element methods for parabolic problems. VI. Analytic semigroups, SIAM J. Numer. Anal. 35 (1998), no. 4, 1315-1325 (electronic), DOI 10.1137/S0036142996310216. MR1620144(99d:65281)

[23] A. Ern and M. Vohralík, A posteriori error estimation based on potential and flux reconstruction for the heat equation, SIAM J. Numer. Anal. 48 (2010), no. 1, 198-223, DOI 10.1137/090759008. MR2608366 (2011d:65281)

[24] X. Feng and O. A. Karakashian, Two-level non-overlapping Schwarz preconditioners for a discontinuous Galerkin approximation of the biharmonic equation, J. Sci. Comput. 22/23 (2005), 289-314, DOI 10.1007/s10915-004-4141-9. MR2142199 (2006b:65156)

[25] X. Feng and O. A. Karakashian, Fully discrete dynamic mesh discontinuous Galerkin methods for the Cahn-Hilliard equation of phase transition, Math. Comp. 76 (2007), no. 259, 10931117 (electronic), DOI 10.1090/S0025-5718-07-01985-0. MR2299767(2008a:74048)

[26] X. Feng and $\mathrm{H}$. Wu, A posteriori error estimates for finite element approximations of the Cahn-Hilliard equation and the Hele-Shaw flow, J. Comput. Math. 26 (2008), no. 6, 767-796. MR2464735 (2009j:35148) 
[27] E. H. Georgoulis and P. Houston, Discontinuous Galerkin methods for the biharmonic problem, IMA J. Numer. Anal. 29 (2009), no. 3, 573-594, DOI 10.1093/imanum/drn015. MR.2520159 (2010g:65209)

[28] E. H. Georgoulis, P. Houston, and J. Virtanen, An a posteriori error indicator for discontinuous Galerkin approximations of fourth-order elliptic problems, IMA J. Numer. Anal. 31 (2011), no. 1, 281-298, DOI 10.1093/imanum/drp023. MR2755946(2012b:65180)

[29] E. H. Georgoulis, O. Lakkis, and J. M. Virtanen, A posteriori error control for discontinuous Galerkin methods for parabolic problems, SIAM J. Numer. Anal. 49 (2011), no. 2, 427-458, DOI 10.1137/080722461. MR2784879(2012d:65205)

[30] P. Grisvard, Elliptic Problems in Nonsmooth Domains, Monographs and Studies in Mathematics, vol. 24, Pitman (Advanced Publishing Program), Boston, MA, 1985. MR775683 (86m:35044)

[31] T. Gudi, Residual-based a posteriori error estimator for the mixed finite element approximation of the biharmonic equation, Numer. Methods Partial Differential Equations 27 (2011), no. 2, 315-328, DOI 10.1002/num.20524. MR2752872 (2012f:65191)

[32] O. A. Karakashian and F. Pascal, Convergence of adaptive discontinuous Galerkin approximations of second-order elliptic problems, SIAM J. Numer. Anal. 45 (2007), no. 2, 641-665 (electronic), DOI 10.1137/05063979X. MR2300291(2008j:65196)

[33] O. Lakkis and C. Makridakis, Elliptic reconstruction and a posteriori error estimates for fully discrete linear parabolic problems, Math. Comp. 75 (2006), no. 256, 1627-1658, DOI 10.1090/S0025-5718-06-01858-8. MR2240628(2007e:65122)

[34] O. Lakkis and T. Pryer, Gradient recovery in adaptive finite-element methods for parabolic problems, IMA J. Numer. Anal. 32 (2012), no. 1, 246-278, DOI 10.1093/imanum/drq019. MR.2875251

[35] S. Larsson, and A. Mesforush, A posteriori error analysis for the Cahn-Hilliard equation, Chalmers University of Technology Preprint 2010:19 (2010).

[36] C. Makridakis and R. H. Nochetto, Elliptic reconstruction and a posteriori error estimates for parabolic problems, SIAM J. Numer. Anal. 41 (2003), no. 4, 1585-1594, DOI 10.1137/S0036142902406314. MR2034895 (2004k:65157)

[37] I. Mozolevski and E. Süli, A priori error analysis for the hp-version of the discontinuous Galerkin finite element method for the biharmonic equation, Comput. Methods Appl. Math. 3 (2003), no. 4, 596-607, DOI 10.2478/cmam-2003-0037. MR2048235(2005c:65106)

[38] P. Neittaanmäki and S. I. Repin, A posteriori error estimates for boundary-value problems related to the biharmonic operator, East-West J. Numer. Math. 9 (2001), no. 2, 157-178. MR.1836871

[39] B. Rivière and M. F. Wheeler, A posteriori error estimates for a discontinuous Galerkin method applied to elliptic problems, Comput. Math. Appl. 46 (2003), no. 1, 141-163, DOI 10.1016/S0898-1221(03)90086-1. MR2015276 (2004j:65169)

[40] R. H. Stogner and G. F. Carey, $C^{1}$ macroelements in adaptive finite element methods, Internat. J. Numer. Methods Engrg. 70 (2007), no. 9, 1076-1095, DOI 10.1002/nme.1912. MR:2322460(2008f:65222)

[41] E. Süli and I. Mozolevski, hp-version interior penalty DGFEMs for the biharmonic equation, Comput. Methods Appl. Mech. Engrg. 196 (2007), no. 13-16, 1851-1863, DOI 10.1016/j.cma.2006.06.014. MR.2298696 (2008c:65350)

[42] R. Verfürth, A Review of A Posteriori Error Estimation and Adaptive Mesh-Refinement Techniques, Wiley-Teubner, Chichester-Stuttgart, 1996.

[43] J. M. Virtanen, Adaptive discontinuous Galerkin methods for fourth order problems, Ph.D. Thesis, University of Leicester (2010).

Department of Mathematics, University of Leicester, University Road, Leicester

LE1 7RH, UNITED KingDOM.

E-mail address: Emmanuil.Georgoulis@le.ac.uk

Department of Mathematics, University of Leicester, University Road, Leicester

LE1 7RH, UNITED KINGDOM

E-mail address: jv77@le.ac.uk 